\title{
Algebraic treatment of super-integrable potentials
}

\author{
L. Chetouani, L. Guechi \\ Département de Physique, \\ Faculté des Sciences, Université Mentouri, \\ Route d'Ain El Bey, Constantine, Algeria. \\ T.F. Hammann \\ Laboratoire de Mathématiques, Physique Mathématique, \\ Faculté des Sciences et Techniques, \\ Université de Haute Alsace, \\ 4, rue des Frères Lumière, F-68093 Mulhouse, France
}

\begin{abstract}
The so $(2,1)$ Lie algebra is applied to three classes of two- and threedimensional Smorodinsky-Winternitz super-integrable potentials for which the path integral discussion has been recently presented in the literature. We have constructed the Green's functions for two important super-integrable potentials in $R^{2}$. Among the super-integrable potentials in $R^{3}$, we have considered two examples, one is maximally super-integrable and another one minimally super-integrable. The discussion is made in various coordinate systems. The energy spectrum and the suitably normalized wave functions of bound and continuous states are then deduced.
\end{abstract}

PACS 03.65-Quantum theory ; quantum mechanics

PACS 03.65.Fd -Algebraic methods 


\section{Introduction}

Ever since the success of the algebraic approach based on the non-compact groups in calculating the wave functions and the transition amplitudes for the hydrogen atom by Kleinert ${ }^{1}$, a renewed interest for this method has been emerging. Hence a large amount of physical problems for which the Schrödinger equation reduces to the confluent hypergeometric equation have been treated in the framework of this approach. In particular, the radial harmonic oscillator, the radial Coulomb and Morse oscillator potentials and the Natanzon confluent potentials ${ }^{2}$, which generalize the latter, have been discussed in a variety of $\mathrm{SO}(2,1)$ realizations $^{3-9}$.

The above so $(2,1)$ algebraic approach has also been applied to noncentral potentials such as the ring-shaped potential ${ }^{10}$ introduced by Hartmann $^{11}$ to describe the molecular interaction of cyclic polyenes and the so-called double ring-shaped oscillator ${ }^{12}$ which is the Quesne ring-shaped oscillator ${ }^{13}$ plus an $r^{-2} \sec ^{2} \theta$ term.

The (MS) variant of the algebraic method summarized in section II has been developed by Milshtein and Strakhovenko ${ }^{14}$ to construct the Green's function associated with the problem of Dirac electron in a static Coulomb field. The straightforward algebraic calculation of the Green's function for a given potential represents an undeniable advantage which allowed this variant to be given a great deal of attention, in recent years, following the development of path integration techniques. Hence a remarkable set of potentials has been studied in this algebraic approach. Among them, we can quote potentials of practical interest, such as Morse's ${ }^{15}$, the radial harmonic oscillator and the radial Coulomb potentials . The Hartmann ring-shaped potential $^{16}$, the compound Coulomb plus Aharonov-Bohm potential ${ }^{17}$ and two highly singular non-isotropic potentials associated to a highly distorted spherical Coulomb field with an additional double ring well and a highly distorted cylindrical Coulomb field have also been studied in parabolic coordinate systems $^{18}$. Within the framework of the $R^{4}$ to $R^{3}$ non-bijective Kustaanheimo-Stiefel mapping, the Kaluza-Klein monopole system ${ }^{19}$ and a noncentral potential ${ }^{20}$ which generalizes the Coulomb potential and the Hartmann ring-shaped potential and also, due to its close link with the latter, the compound Coulomb plus Aharonov-Bohm potential have been treated by means of the same algebraic approach as Milshtein and Strakhovenko. 
Cylindrical parabolic coordinates have also been used in discussion for the so $(2,1)$ algebraic method of another type of noncentral potentials ${ }^{21,22}$. All these potentials discussed with the help of the (MS) variant appear in the general classification of potentials in two and three dimensions possessing dynamical invariance groups initiated about 30 years ago by Smorodinsky and co-workers ${ }^{23}$, continued by Kibler and Winternitz ${ }^{24}$, and revived, in recent years, by Evans ${ }^{25,26}$. This classification was established according to the number of degrees of freedom, quadratic integrals of motion in the momenta and coordinate systems in which the potential allows the separation of variables. The Hamiltonian systems with these potentials are called super-integrable. Generally, in $n$ dimensions, a system is called "minimally" super-integrable if it has $(2 n-2)$ constants or integrals of motion ( including energy ), and it is called "maximally" super-integrable if it has $(2 n-1)$ integrals of motion ${ }^{27}$. A list of minimally super-integrable and maximally super-integrable potentials with the corresponding constants of motion in the classical form and all separating coordinate systems has been established by Evans $^{28}$. On the basis of this classification, Grosche et $\mathrm{al}^{29}$ have recently presented a detailed path integral discussion of the so-called SmorodinskyWinternitz super-integrable potentials in many coordinate systems. It is to be noted that almost all the potentials contained in this classification involve centrifugal or angular barriers which possess point singularities. Consequently, following Kleinert ${ }^{30}$ the time sliced path integral for these potentials does not exist in any coordinate system. So, it is necessary to regularize the system in question by an appropriate set of new coordinates in order to find a path integral expression without collapse. This problem does not occur in the framework of the (MS) variant of the so $(2,1)$ algebraic approach owing to its local (differential) character. This constitutes a great advantage in studying the problem of the Smorodinsky-Winternitz potentials in this framework.

The plan of this article is as follows. We briefly review the so $(2,1)$ Lie algebra and its use in calculating Green's functions in section II. We study a set of two potentials in two dimensions, algebraically obtain the Green's function in the various separating coordinate systems and deduce the energy spectrum as well as the corresponding normalized wave functions in section III. Sections IV and V deal with two examples of three-dimensional potentials. The construction of the Green's functions is made in different coordinate systems. The energy spectrum and the normalized wave functions 
are evaluated. Section VI will be a conclusion.

\section{Green's function and so(2,1) Lie algebra}

Let's briefly review the main features of so $(2,1)$ Lie algebra and its use in the calculation of the Green's functions to make this paper self-contained. A set of three operators $\left\{T_{1}, T_{2}, T_{3}\right\}$ characterized by the commutation relations $^{31}$ define it:

$$
\left[T_{1}, T_{2}\right]=-i T_{1}, \quad\left[T_{2}, T_{3}\right]=-i T_{3}, \quad\left[T_{1}, T_{3}\right]=-i T_{2} .
$$

Because of the type of potentials we shall deal with in this paper, we have to use the following differential realization of the operators:

$$
T_{1}(x)=-\frac{\hbar^{2}}{2 M}\left(\frac{\partial^{2}}{\partial x^{2}}-\frac{\mu(\mu-1)}{x^{2}}\right), T_{2}(x)=-\frac{i}{2}\left(x \frac{\partial}{\partial x}+\frac{1}{2}\right), T_{3}(x)=\frac{M}{4 \hbar^{2}} x^{2},
$$

with $0<x<\infty$.

By using Schwinger's integral representation ${ }^{32}$, the Green's function associated to a potential $V(x)$ with the $\mathrm{SO}(2,1)$ group symmetry is given by

$$
G\left(x, x^{\prime} ; E\right)=\int_{0}^{\infty} d S \exp \left[\frac{i S}{\hbar}(E+i 0)\right] K\left(x, x^{\prime} ; S\right),
$$

where

$$
\begin{aligned}
K\left(x, x^{\prime} ; S\right) & =\exp \left\{-\frac{i S}{\hbar}\left[-\frac{\hbar^{2}}{2 M} \nabla_{x}^{2}+V(x)\right]\right\} \delta\left(x-x^{\prime}\right) \\
& =\exp \left\{-\frac{i S}{\hbar}\left[T_{1}(x)+2 \hbar^{2} \omega^{2} T_{3}(x)\right]\right\} \delta\left(x-x^{\prime}\right) .
\end{aligned}
$$

The calculation of this kernel is based upon the use of two Baker-CampbellHausdorff formulas ${ }^{33}$

$$
\exp \left\{-\frac{i S}{\hbar}\left[T_{1}+2 \hbar^{2} \omega^{2} T_{3}\right]\right\}=\exp \left(-i a T_{3}\right) \exp \left(-i b T_{2}\right) \exp \left(-i c T_{1}\right),
$$


where

$$
a=2 \hbar \omega \tan (\omega S), \quad b=2 \operatorname{Ln}[\cos (\omega S)], \quad c=\frac{1}{\hbar \omega} \tan (\omega S),
$$

and

$$
\exp \left(-i \alpha T_{3}\right) \exp \left(-i \beta T_{2}\right) \exp \left(-i \gamma T_{1}\right)=\exp \left(-i c T_{1}\right) \exp \left(\tau T_{3}\right),
$$

with

$$
\alpha=\frac{i \tau}{1-i \tau c / 2}, \quad \beta=2 \operatorname{Ln}\left(1-\frac{i \tau c}{2}\right), \quad \gamma=\frac{c}{1-i \tau c / 2} .
$$

Here, we also have to use the Laplace transform of the Dirac distribution

$$
\delta\left(x-x^{\prime}\right)=\frac{M}{2 \hbar^{2}} \frac{x^{\mu} x^{1-\mu}}{2 i \pi} \int_{-i \infty+\delta}^{i \infty+\delta} d \tau \exp \left[\frac{M}{4 \hbar^{2}}\left(x^{2}-x^{\prime 2}\right) \tau\right] ; \quad \delta<0,
$$

in order to obtain a manageable result as follows:

$$
\exp \left(-i \gamma T_{1}\right) x^{\mu}=\left[1-i \gamma T_{1}+\frac{1}{2 !}\left(-i \gamma T_{1}\right)^{2}+\ldots\right] x^{\mu}=x^{\mu} .
$$

Using relations (9), (5) and (7), the kernel (4) can now be written

$$
\begin{aligned}
K\left(x, x^{\prime} ; S\right)= & \frac{M}{2 \hbar^{2}} x^{1-\mu} \exp \left(-i a T_{3}\right) \exp \left(-i b T_{2}\right) x^{\mu} \exp \left(\frac{i M x^{2}}{2 \hbar^{2} c}\right) \\
& \times \int_{-i \infty+\delta}^{i \infty+\delta} d \tau \frac{\exp \left(-\frac{M x^{2}}{4 \hbar^{2}} \tau\right) \exp \left(\frac{M x^{2}}{\hbar^{2} c^{2}} \frac{1}{\tau+2 i / c}\right)}{\left(1-\frac{i \tau c}{2}\right)^{\mu+\frac{1}{2}}},
\end{aligned}
$$

where the well-known formula

$$
\exp \left(-i \beta T_{2}\right) f(x)=\exp \left(-\frac{\beta}{4}\right) f\left(e^{-\frac{\beta}{2}} x\right)
$$

has also been used.

The integral can be calculated thanks to the residue theorem after the $\exp \left(\frac{M x^{2}}{\hbar^{2} c^{2}} \frac{1}{\tau+2 i / c}\right)$ series has been effected. Hence, we obtain

$$
\begin{aligned}
K\left(x, x^{\prime} ; S\right)= & \frac{M \omega}{i \hbar \sin (\omega S)} \sqrt{x x^{\prime}} \exp \left[\frac{i M \omega}{2 \hbar}\left(x^{2}+x^{\prime 2}\right) \cot (\omega S)\right] \\
& \times I_{\lambda}\left(\frac{M \omega x x^{\prime}}{i \hbar \sin (\omega S)}\right),
\end{aligned}
$$


where $\lambda=\mu-\frac{1}{2}$ and $I_{\lambda}(x)$ is the modified Bessel function.

We can now use (13) for any coordinate in a multi-dimensional system, provided that the $(H-E)^{-1}$ inverse resolvent operator can be transformed into a linear combination of the above mentioned $T_{i}(i=1,2,3)$ operators.

\section{Two-dimensional maximally super-integrable po- tentials}

We shall study here a set of two important potentials belonging to a class of two-dimensional Smorodinsky-Winternitz potentials. They are characterized by the existence of three functionally independent integrals of motion, which means that there is a pair of quadratic operators corresponding to these integrals of motion which commute with the system's Hamiltonian. The number of such integrals being superior to that of degrees of liberty, they are thus called maximally super-integrable potentials.

\section{A. Let us study the potential}

$$
V_{1}(\vec{\rho})=-\frac{\alpha_{0}}{\sqrt{x_{1}^{2}+x_{2}^{2}}}+\frac{\hbar^{2}}{4 M} \frac{1}{\sqrt{x_{1}^{2}+x_{2}^{2}}}\left(\frac{k_{1}^{2}-\frac{1}{4}}{\sqrt{x_{1}^{2}+x_{2}^{2}}+x_{1}}+\frac{k_{2}^{2}-\frac{1}{4}}{\sqrt{x_{1}^{2}+x_{2}^{2}}-x_{1}}\right),
$$

with positive $\alpha_{0}, k_{1}$ and $k_{2}$ constants . It admits the following three functionally independent integrals of motion:

$$
\left\{\begin{array}{l}
H_{1}=\frac{P^{2}}{2 M}+V_{1}(\vec{\rho}), \quad I_{1}=\frac{L_{3}^{2}}{2 M}+\frac{\hbar^{2} \rho^{2}}{8 M}\left(\frac{k_{1}^{2}-\frac{1}{4}}{x_{1}^{2}}+\frac{k_{2}^{2}-\frac{1}{4}}{x_{2}^{2}}\right) \\
I_{2}=\frac{\left\{L_{3}, P_{2}\right\}}{4 M}+\frac{\alpha(\eta-\xi)}{\xi \eta}-\frac{2\left(k_{1}^{2}-\frac{1}{4}\right)}{\xi^{2}}+\frac{2\left(k_{2}^{2}-\frac{1}{4}\right)}{\eta^{2}} .
\end{array}\right.
$$

This potential is exactly solvable in two coordinate systems, namely parabolic and polar. For $k_{1}=k_{2}=\frac{1}{2}$, equation (14) can be reduced to the Coulomb potential treated with a path integral approach ${ }^{34,35}$. The algebraic solution to this potential via the (MS) variant is easier to establish

using the parabolic coordinates or even the Levi-Cevita variables ${ }^{36}$ defined 
by $x_{1}=u_{1}^{2}-u_{2}^{2}, \quad x_{2}=2 u_{1} u_{2}\left(-\infty<u_{1}, u_{2}<+\infty\right)$. The Green's func-

tion $G(\vec{\rho}, \vec{\rho} ; E)$ associated to the potential (14), in Schwinger's integral representation, is given by

$$
\begin{aligned}
G\left(\vec{\rho}, \overrightarrow{\rho^{\prime}} ; E\right)= & \int_{0}^{\infty} \frac{d S}{4 \rho} \exp \left\{\frac { i S } { \hbar } \left[\frac{1}{4 \rho} \sum_{j=1}^{2}\left(-\frac{\hbar^{2}}{2 M}\left(\frac{\partial^{2}}{\partial u_{j}^{2}}-\frac{k_{j}^{2}-\frac{1}{4}}{u_{j}^{2}}\right)\right)\right.\right. \\
& \left.\left.-\frac{\alpha_{0}}{\rho}-E-i 0\right]\right\} \prod_{j=1}^{2} \delta\left(u_{j}-u_{j}^{\prime}\right) .
\end{aligned}
$$

By applying the time transformation $S \rightarrow \tau$ defined by $\tau=\frac{S}{4 \rho}$, the Green's function (16) can be written

$$
G\left(\vec{\rho}, \overrightarrow{\rho^{\prime}} ; E\right)=\int_{0}^{\infty} d \tau \exp \left[\frac{i}{\hbar}\left(4 \alpha_{0}+i 0\right) \tau\right] \prod_{j=1}^{2} K\left(u_{j}, u_{j}^{\prime} ; \tau\right),
$$

where

$$
K\left(u_{j}, u_{j}^{\prime} ; \tau\right)=\exp \left\{-\frac{i \tau}{\hbar}\left[T_{1}\left(u_{j}\right)+2 \hbar^{2} \omega^{2} T_{3}\left(u_{j}\right)\right]\right\} \delta\left(u_{j}-u_{j}^{\prime}\right),
$$

with $\mu_{j}=\frac{1}{2} \pm k_{j}$ and $\omega=\sqrt{-\frac{8 E}{M}}$.

Using the equation (13), we can write the propagators (18) as follows:

$$
K\left(u_{j}, u_{j}^{\prime} ; \tau\right)=\frac{M \omega \sqrt{u_{j} u_{j}^{\prime}}}{i \hbar \sin (\omega \tau)} \exp \left[\frac{i M \omega}{2 \hbar}\left(u_{j}^{2}+u_{j}^{\prime 2}\right) \cot (\omega \tau)\right] I_{ \pm k_{j}}\left(\frac{M \omega u_{j} u_{j}^{\prime}}{i \hbar \sin (\omega \tau)}\right) .
$$

\section{Parabolic coordinates}

In the parabolic coordinates $\xi=\sqrt{2} u_{1}, \eta=\sqrt{2} u_{2}$ and after changing $\omega \rightarrow 2 \omega$ and $\tau \rightarrow \frac{\tau}{2}$ and by taking into account (19), the Green's function (17) becomes

$$
G\left(\vec{\rho}, \overrightarrow{\rho^{\prime}} ; E\right)=\int_{0}^{\infty} d \tau \exp \left[\frac{i}{\hbar}\left(2 \alpha_{0}+i 0\right) \tau\right] K\left(\xi, \eta, \xi^{\prime}, \eta^{\prime} ; \tau\right),
$$


where

$$
\begin{aligned}
K\left(\xi, \eta, \xi^{\prime}, \eta^{\prime} ; \tau\right)= & \left(\frac{M \omega}{i \hbar \sin (\omega \tau)}\right)^{2} \sqrt{\xi \xi^{\prime} \eta \eta^{\prime}} \\
& \times \exp \left[\frac{i M \omega}{2 \hbar}\left(\xi^{2}+\eta^{2}+\xi^{\prime 2}+\eta^{\prime 2}\right) \cot (\omega \tau)\right] \\
& \times I_{ \pm k_{1}}\left(\frac{M \omega \xi \xi^{\prime}}{i \hbar \sin (\omega \tau)}\right) I_{ \pm k_{2}}\left(\frac{M \omega \eta \eta^{\prime}}{i \hbar \sin (\omega \tau)}\right),
\end{aligned}
$$

with

$$
\omega=\sqrt{-\frac{2 E}{M}} .
$$

To find the energy spectrum and the normalized wave functions of the bound states, we make use the Hille and Hardy formula ${ }^{37}$

$$
\frac{1}{1-z}\left[-z \frac{x+y}{1-z}\right] I_{\alpha}\left(\frac{2 \sqrt{x y z}}{1-z}\right)=(x y z)^{\frac{\alpha}{2}} \sum_{n=0}^{\infty} \frac{n !}{\Gamma(n+\alpha+1)} L_{n}^{\alpha}(x) L_{n}^{\alpha}(y) z^{n} ;|z|<1,
$$

where the $L_{n}^{\alpha}(x)$ are the Laguerre polynomials, and the integration over $S$ yields the quantization condition

$$
n_{1}+n_{2} \pm \frac{k_{1}}{2} \pm \frac{k_{2}}{2}+p=0 .
$$

where $p=-\frac{\alpha_{0}}{\hbar \omega}$. Therefore, the Green's function (20) can be written as:

$$
G\left(\vec{\rho}, \overrightarrow{\rho^{\prime}} ; E\right)=i \hbar \sum_{n_{1}, n_{2}=0}^{\infty} \frac{\Psi_{n_{1}, n_{2}}(\xi, \eta) \Psi_{n_{1}, n_{2}}^{*}\left(\xi^{\prime}, \eta^{\prime}\right)}{E+i 0-E_{n_{1}, n_{2}}},
$$

with the normalized wave functions ( $a=\frac{\hbar^{2}}{M \alpha_{0}}$ is the Bohr radius)

$$
\begin{aligned}
\Psi_{n_{1}, n_{2}}(\xi, \eta)= & {\left[\frac{2}{a^{2} N^{3}} \frac{n_{1} ! n_{2} !}{\Gamma\left(n_{1} \pm k_{1}+1\right) \Gamma\left(n_{2} \pm k_{2}+1\right)}\right]^{\frac{1}{2}}\left(\frac{\xi^{2}}{a N}\right)^{\frac{1}{4} \pm \frac{k_{1}}{2}} } \\
& \left(\frac{\eta^{2}}{a N}\right)^{\frac{1}{4}} \frac{k_{2}}{2} \exp \left[-\frac{\xi^{2}+\eta^{2}}{2 a N}\right] L_{n_{1}}^{ \pm k_{1}}\left(\frac{\xi^{2}}{a N}\right) L_{n_{2}}^{ \pm k_{2}}\left(\frac{\eta^{2}}{a N}\right),
\end{aligned}
$$


and the discrete energy spectrum given by

$$
E_{n_{1}, n_{2}}=-\frac{M \alpha_{0}^{2}}{2 \hbar^{2} N^{2}} ; \quad N=n_{1}+n_{2} \pm \frac{k_{1}}{2} \pm \frac{k_{2}}{2}+1 .
$$

To determine the energy spectrum and the wave functions of the continuous states, let's go back to the expression (21) and use the dispersion formula (see Ref. ${ }^{37}$, p. 884, Eq. (7.694))

$$
\begin{aligned}
& \frac{2 \pi \sqrt{x y}}{\sin \alpha} \exp [-(x+y) \cot \alpha] I_{2 \mu}\left(\frac{2 \sqrt{x y}}{\sin \alpha}\right) \\
= & \int_{R} d p \frac{\Gamma\left(\frac{1}{2}+\mu+i p\right) \Gamma\left(\frac{1}{2}+\mu-i p\right)}{\Gamma^{2}(2 \mu+1)} M_{i p, \mu}(-2 i x) M_{-i p, \mu}(2 i y) .
\end{aligned}
$$

Given $x=\frac{M \omega}{2 i \hbar} \xi^{\prime 2}, y=\frac{M \omega}{2 i \hbar} \xi^{2}$ and $\alpha=\omega \tau$, we obtain

$$
\begin{aligned}
K\left(\xi, \eta, \xi^{\prime}, \eta^{\prime} ; \tau\right)= & \frac{1}{\sqrt{\xi \xi^{\prime} \eta \eta^{\prime}} \pi^{2} \Gamma^{2}\left(1 \pm k_{1}\right) \Gamma^{2}\left(1 \pm k_{2}\right)} \int_{-\infty}^{\infty} \int_{-\infty}^{\infty} d p_{\xi} d p_{\eta} \\
& \times \Gamma\left(\frac{1}{2} \pm \frac{k_{1}}{2}+i p_{\xi}\right) \Gamma\left(\frac{1}{2} \pm \frac{k_{1}}{2}-i p_{\xi}\right) \\
& \times \Gamma\left(\frac{1}{2} \pm \frac{k_{2}}{2}+i p_{\eta}\right) \Gamma\left(\frac{1}{2} \pm \frac{k_{2}}{2}-i p_{\eta}\right) e^{(\pi-2 \omega \tau)\left(p_{\xi}+p_{\eta}\right)} \\
& \times M_{-i p_{\xi}, \pm \frac{k_{1}}{2}}\left(\frac{M \omega}{\hbar} \xi^{2}\right) M_{i p_{\xi}, \pm \frac{k_{1}}{2}}\left(\frac{M \omega}{\hbar} \xi^{\prime 2}\right) \\
& \times M_{-i p_{\eta}, \pm \frac{k_{2}}{2}}\left(\frac{M \omega}{\hbar} \eta^{2}\right) M_{i p_{\eta}, \pm \frac{k_{2}}{2}}\left(\frac{M \omega}{\hbar} \eta^{\prime 2}\right)
\end{aligned}
$$

If we now transfer (29) into (20), we shall obtain the poles of the continuous state Green's function by integration on the $\tau$ variable. They will be defined by

$$
\omega\left(p_{\xi}+p_{\eta}\right)-\frac{i \alpha_{0}}{\hbar}=0 .
$$

If we convert this into energy via (22), the values of the energy will be

$$
E_{p}=\frac{\hbar^{2} p^{2}}{2 M} \quad \text { with } \quad p=\frac{1}{a\left(p_{\xi}+p_{\eta}\right)} .
$$


Let's now change the variables defined by

$$
p_{\xi}=\frac{1}{2 p}\left(\frac{1}{a}+\varsigma\right) \quad \text { and } \quad p_{\eta}=\frac{1}{2 p}\left(\frac{1}{a}-\varsigma\right) .
$$

In this case, we can write the Green's function as follows:

$$
G^{c}\left(\xi, \eta, \xi^{\prime}, \eta^{\prime} ; E\right)=i \hbar \int_{0}^{\infty} d p \int_{-\infty}^{\infty} d \varsigma \frac{\Psi_{p, \varsigma}(\xi, \eta) \Psi_{p, \varsigma}^{*}\left(\xi^{\prime}, \eta^{\prime}\right)}{E+i 0-E_{p}},
$$

with the wave functions given by

$$
\begin{aligned}
\Psi_{p, \varsigma}(\xi, \eta)= & \frac{\left|\Gamma\left(\frac{1}{2} \pm \frac{k_{1}}{2}+\frac{i}{2 p}\left(\frac{1}{a}+\varsigma\right)\right) \Gamma\left(\frac{1}{2} \pm \frac{k_{2}}{2}+\frac{i}{2 p}\left(\frac{1}{a}-\varsigma\right)\right)\right|}{2 \pi \Gamma\left(1 \pm k_{1}\right) \Gamma\left(1 \pm k_{2}\right) \sqrt{\xi \eta}} \frac{e^{\frac{\pi}{2 a p}}}{\sqrt{p}} \\
& \times M_{-\frac{i}{2 p}\left(\frac{1}{a}+\varsigma\right), \pm \frac{k_{1}}{2}}\left(-i p \xi^{2}\right) M_{-\frac{i}{2 p}\left(\frac{1}{a}-\varsigma\right), \pm \frac{k_{2}}{2}}\left(-i p \eta^{2}\right)
\end{aligned}
$$

\section{Polar coordinates}

Let's use the $(\rho, \phi)$ polar coordinates defined by

$$
\xi=\sqrt{2} u_{1}=\sqrt{2 \rho} \cos \frac{\phi}{2}, \eta=\sqrt{2} u_{2}=\sqrt{2 \rho} \sin \frac{\phi}{2},
$$

and the addition theorem formula ${ }^{38}$ :

$$
\begin{aligned}
& \frac{z}{2} I_{\nu}(z \sin \alpha \sin \beta) I_{\mu}(z \cos \alpha \cos \beta) \\
= & (\sin \alpha \sin \beta)^{\nu}(\cos \alpha \cos \beta)^{\mu} \sum_{n=0}^{\infty}(\nu+\mu+2 n+1) \frac{n !}{\Gamma(\nu+n+1)} \\
& \times \frac{\Gamma(\nu+\mu+n+1)}{\Gamma(\mu+n+1)} I_{\mu+\nu+2 n+1}(z) P_{n}^{(\nu, \mu)}(\cos (2 \alpha)) P_{n}^{(\nu, \mu)}(\cos (2 \beta)) .
\end{aligned}
$$

This will give us the following form of the Green's function (20):

$$
G\left(\vec{\rho}, \overrightarrow{\rho^{\prime}} ; E\right)=\sum_{n=0}^{\infty} G_{n}\left(\rho, \rho^{\prime} ; E\right) \Phi_{n}^{\left( \pm k_{2}, \pm k_{1}\right)}\left(\frac{\phi}{2}\right) \Phi_{n}^{\left( \pm k_{2}, \pm k_{1}\right)}\left(\frac{\phi^{\prime}}{2}\right)
$$


where the angular wave functions are those defined in function of the $P_{n}^{\left( \pm k_{2}, \pm k_{1}\right)}(\cos \phi)$ Jacobi polynomials by

$$
\begin{aligned}
\Phi_{n}^{\left( \pm k_{2}, \pm k_{1}\right)}(\phi)= & {\left[2\left(2 n \pm k_{1} \pm k_{2}+1\right) \frac{n ! \Gamma\left(n \pm k_{1} \pm k_{2}+1\right)}{\Gamma\left(n \pm k_{1}+1\right) \Gamma\left(n \pm k_{2}+1\right)}\right]^{\frac{1}{2}} } \\
& \times(\sin \phi)^{\frac{1}{2} \pm k_{2}}(\cos \phi)^{\frac{1}{2} \pm k_{1}} P_{n}^{\left( \pm k_{2}, \pm k_{1}\right)}(\cos (2 \phi)) .
\end{aligned}
$$

The radial Green's function $G_{n}\left(\rho, \rho^{\prime} ; E\right)$ included in (37) is defined by

$$
\begin{aligned}
G_{n}\left(\rho, \rho^{\prime} ; E\right)= & \frac{M \omega}{i \hbar} \int_{0}^{\infty} \frac{d \tau}{\sin (\omega \tau)} \exp \left[\frac{i}{\hbar}\left(2 \alpha_{0}+i 0\right) \tau\right] \\
& \times \exp \left[\frac{i M \omega}{2 \hbar}\left(\rho+\rho^{\prime}\right) \cot (\omega \tau)\right] I_{2 \lambda}\left(\frac{2 M \omega \sqrt{\rho \rho^{\prime}}}{i \hbar \sin (\omega \tau)}\right),
\end{aligned}
$$

with $\lambda=n+\frac{1}{2}\left(1 \pm k_{1} \pm k_{2}\right)$.

Then, thanks to formula (see Ref. ${ }^{37}$, p. 729, Eq. (6.699.4))

$$
\begin{aligned}
& \int_{0}^{\infty} d q \frac{e^{-2 p q}}{\sinh q} \exp \left[-\frac{1}{2}(x+y) \operatorname{coth} q\right] I_{2 \gamma}\left(\frac{\sqrt{x y}}{\sinh q}\right) \\
= & \frac{\Gamma\left(p+\gamma+\frac{1}{2}\right)}{\Gamma(2 \gamma+1) \sqrt{x y}} M_{-p, \gamma}(x) W_{-p, \gamma}(y),
\end{aligned}
$$

valid for $\operatorname{Re}\left(p+\gamma+\frac{1}{2}\right)>0, \operatorname{Re}(\gamma)>0$ and $y>x$, where $M_{-p, \gamma}(x)$ and $W_{-p, \gamma}(y)$ are the Whittaker functions, we can write (39) as follows:

$$
G_{n}\left(\rho, \rho^{\prime} ; E\right)=\frac{\Gamma\left(p+\lambda+\frac{1}{2}\right)}{2 i \omega \Gamma(2 \lambda+1) \sqrt{\rho \rho^{\prime}}} M_{-p, \lambda}\left(\frac{2 M \omega}{\hbar} \rho^{\prime}\right) W_{-p, \lambda}\left(\frac{2 M \omega}{\hbar} \rho\right)
$$

with $p=-\frac{\alpha_{0}}{\hbar \omega}, \omega=\sqrt{-\frac{2 E}{M}}$ and $\rho>\rho^{\prime}$.

From (41) and (37), we can deduce that the complete Green's function is given by

$$
\begin{aligned}
G\left(\vec{\rho}, \overrightarrow{\rho^{\prime}} ; E\right)= & \sum_{n=0}^{\infty} \Phi_{n}^{\left( \pm k_{2}, \pm k_{1}\right)}\left(\frac{\phi}{2}\right) \Phi_{n}^{\left( \pm k_{2}, \pm k_{1}\right)}\left(\frac{\phi^{\prime}}{2}\right) \frac{\Gamma\left(p+\lambda+\frac{1}{2}\right)}{2 i \omega \Gamma(2 \lambda+1) \sqrt{\rho \rho^{\prime}}} \\
& \times M_{-p, \lambda}\left(\frac{2 M \omega}{\hbar} \rho^{\prime}\right) W_{-p, \lambda}\left(\frac{2 M \omega}{\hbar} \rho\right) .
\end{aligned}
$$


The normalized wave functions and the energy spectrum of the bound states are given by expression (23) applied to $G_{n}\left(\rho, \rho^{\prime} ; E\right)$ as defined in (39) provided we make the adequate change of variables and thus obtain:

$$
\begin{aligned}
& \Psi_{m, n}(\rho, \phi)= {\left[\frac{m !}{a^{2}\left(m+\lambda+\frac{1}{2}\right)^{3} \Gamma(m+2 \lambda+1)}\right]^{\frac{1}{2}}\left(\frac{2 \rho}{a\left(m+\lambda+\frac{1}{2}\right)}\right)^{\lambda} } \\
& \times \exp \left(-\frac{\rho}{a\left(m+\lambda+\frac{1}{2}\right)}\right) L_{m}^{2 \lambda}\left(\frac{2 \rho}{a\left(m+\lambda+\frac{1}{2}\right)}\right) \\
& \times \Phi_{n}^{\left( \pm k_{2}, \pm k_{1}\right)}\left(\frac{\phi}{2}\right), \\
& E_{m, n}=-\frac{M \alpha_{0}^{2}}{2 \hbar^{2}\left(m+\lambda+\frac{1}{2}\right)^{2}} .
\end{aligned}
$$

In order to evaluate the contribution of the continuous spectrum to the Green's function, let's write (41) as follows:

$$
\begin{aligned}
G_{n}\left(\rho, \rho^{\prime} ; E\right)= & \frac{i \hbar}{4 \pi \Gamma(2 \lambda+1) \sqrt{\rho \rho^{\prime}}} \oint_{C} \frac{d z}{E+i 0-\frac{\hbar^{2} z^{2}}{2 M}} \Gamma\left(p+\lambda+\frac{1}{2}\right) \\
& \times M_{-p, \lambda}\left(\frac{2 M \omega}{\hbar} \rho^{\prime}\right) W_{-p, \lambda}\left(\frac{2 M \omega}{\hbar} \rho\right),
\end{aligned}
$$

where $C$ is the closed contour,

$$
C:\left\{\begin{array}{c}
z=k ; k \in[-R, R] \\
z=\operatorname{Re}^{i \phi}, \phi \in(\pi, 2 \pi) .
\end{array}\right.
$$

At the $R \rightarrow \infty$ limit, taking the asymptotic behaviour of the Whittaker functions (see Ref. ${ }^{37}$, p. 1061, Eq. (9.227)) into account, it is easy to demonstrate that the integral over the semicircle vanishes. Which leads to:

$$
\begin{aligned}
G_{n}\left(\rho, \rho^{\prime} ; E\right)= & \frac{i \hbar}{4 \pi \Gamma(2 \lambda+1) \sqrt{\rho \rho^{\prime}}} \int_{-\infty}^{\infty} \frac{d k}{E+i 0-\frac{\hbar^{2} k^{2}}{2 M}} \Gamma\left(p+\lambda+\frac{1}{2}\right) \\
& \times M_{-p, \lambda}\left(-2 i k \rho^{\prime}\right) W_{-p, \lambda}(-2 i k \rho) .
\end{aligned}
$$

By using the following formulas (see Ref. ${ }^{37}$, pp. 1061-2, Eqs. (9.231.2) and $(9.233 .1))$

$$
M_{\lambda, \mu}(z)=e^{-i \pi\left(\mu+\frac{1}{2}\right)} M_{-\lambda, \mu}(-z), \quad \text { with } \quad 2 \mu \neq-1,-2,-3, \ldots,
$$


and

$$
M_{\lambda, \mu}(z)=\Gamma(2 \mu+1) e^{i \pi \lambda}\left[\frac{W_{-\lambda, \mu}(-z)}{\Gamma\left(\mu-\lambda+\frac{1}{2}\right)}+e^{-i \pi\left(\mu+\frac{1}{2}\right)} \frac{W_{\lambda, \mu}(z)}{\Gamma\left(\mu+\lambda+\frac{1}{2}\right)}\right],
$$

valid for $\arg z \in]-\frac{3 \pi}{2}, \frac{\pi}{2}[$, and $2 \mu \neq-1,-2,-3, \ldots$, the expression (45) can be written

$$
G_{n}\left(\rho, \rho^{\prime} ; E\right)=\frac{i \hbar}{4 \pi \Gamma^{2}(2 \lambda+1) \sqrt{\rho \rho^{\prime}}} \int_{0}^{\infty} d E_{k} \frac{\Psi_{k, n}(\rho) \Psi_{k, n}^{*}\left(\rho^{\prime}\right)}{E+i 0-E_{k}},
$$

where $E_{k}=\frac{\hbar^{2} k^{2}}{2 M}$ and the radial wave functions given by

$$
\Psi_{k, n}(\rho)=\left(\frac{M}{4 \pi \hbar^{2} k}\right)^{\frac{1}{2}} \frac{\left|\Gamma\left(p+\lambda+\frac{1}{2}\right)\right|}{\Gamma(2 \lambda+1)} \frac{e^{\frac{i \pi p}{2}}}{\sqrt{\rho}} M_{-p, \lambda}(-2 i k \rho),
$$

with $p=-\frac{i}{a k}$.

\section{B. Let us now study the potential}

Let us now study the potential

$$
V_{2}(\vec{\rho})=\frac{\alpha_{0}}{\sqrt{x_{1}^{2}+x_{2}^{2}}}+\frac{\beta_{1} \sqrt{\sqrt{x_{1}^{2}+x_{2}^{2}}+x_{1}}+\beta_{2} \sqrt{\sqrt{x_{1}^{2}+x_{2}^{2}}-x_{1}}}{\sqrt{x_{1}^{2}+x_{2}^{2}}},
$$

with real $\beta_{1}$ and $\beta_{2}$ constants. This potential has the following three functionally independent integrals of motion:

$$
\left\{\begin{array}{c}
H_{2}=\frac{P^{2}}{2 M}+V_{2}(\vec{\rho}), \quad I_{1}=\frac{\left\{L_{3}, P_{1}\right\}}{4 M}-\frac{\alpha(\xi-\eta)+\beta_{1} \eta \sqrt{\xi / 2}-\beta_{2} \xi \sqrt{\eta / 2}}{\xi+\eta} \\
I_{2}=\frac{\left\{L_{3}, P_{1}\right\}}{4 M}-\frac{\alpha(\xi-\eta)+\left(\beta_{1}+\beta_{2}\right)(\eta \sqrt{\xi / 2}-\xi \sqrt{\eta / 2})}{\xi+\eta}
\end{array}\right.
$$

For given parabolic coordinates $x_{1}=\frac{1}{2}\left(\xi^{2}-\eta^{2}\right), \quad x_{2}=\xi \eta,(\xi>0 \quad$ and $\eta \in R$ ), in the Schwinger's integral representation, the Green's function associated to this potential can be expressed

$$
\begin{aligned}
G\left(\vec{\rho}, \overrightarrow{\rho^{\prime}} ; E\right)= & \int_{0}^{\infty} d S \exp \left[\frac{i S}{\hbar}(E+i 0)\right] \\
& \times \exp \left[-\frac{i S}{\hbar} H_{2}(\xi, \eta)\right] \frac{\delta\left(\xi-\xi^{\prime}\right) \delta\left(\eta-\eta^{\prime}\right)}{2 \rho},
\end{aligned}
$$


where

$$
H_{2}(\xi, \eta)=-\frac{\hbar^{2}}{2 M} \frac{1}{2 \rho}\left(\frac{\partial^{2}}{\partial \xi^{2}}+\frac{\partial^{2}}{\partial \eta^{2}}\right)-\frac{\alpha_{0}}{\rho}+\frac{1}{\rho}\left(\beta_{1} \xi+\beta_{2} \eta\right),
$$

with $\rho=\frac{1}{2}\left(\xi^{2}+\eta^{2}\right)$.

If we now perform the time transformation defined by $\sigma=\frac{S}{2 \rho}=\frac{S}{\xi^{2}+\eta^{2}}$ to separate the $\xi$ and $\eta$ variables and use the mutually orthogonal parabolic coordinates $(\widetilde{\xi}, \widetilde{\eta}) \rightarrow\left(\xi-\frac{\beta_{1}}{E}, \eta-\frac{\beta_{2}}{E}\right)$, we arrive at

$$
G\left(\vec{\rho}, \overrightarrow{\rho^{\prime}} ; E\right)=\int_{0}^{\infty} d \sigma \exp \left[\frac{i \sigma}{\hbar}\left(2 \alpha_{0}-\frac{\beta_{1}^{2}+\beta_{2}^{2}}{E}+i 0\right)\right] K\left(\widetilde{\xi}, \widetilde{\xi}^{\prime} ; \sigma\right) K\left(\widetilde{\eta}, \widetilde{\eta}^{\prime} ; \sigma\right),
$$

where each of kernels $K\left(\widetilde{\xi}, \widetilde{\xi}^{\prime} ; \sigma\right)$ and $K\left(\widetilde{\eta}, \widetilde{\eta}^{\prime} ; \sigma\right)$ can be treated with the so $(2,1)$ Lie algebra and we have

$$
\begin{aligned}
K\left(x, x^{\prime} ; \sigma\right) & =\frac{1}{2} \sum_{\mu=0}^{1} \exp \left\{-\frac{i \sigma}{\hbar}\left[T_{1}(x)+2 \hbar^{2} \omega^{2} T_{3}(x)\right]\right\} \delta\left(x-x^{\prime}\right) \\
& =\sqrt{\frac{M \omega}{2 i \pi \hbar \sin (\omega \sigma)}} \exp \left\{\frac{i M \omega}{2 \hbar}\left[\left(x^{2}+x^{\prime 2}\right) \cot (\omega \sigma)-\frac{2 x x^{\prime}}{\sin (\omega \sigma)}\right]\right\},
\end{aligned}
$$

with $x \equiv(\widetilde{\xi}$ or $\widetilde{\eta})$ and $\omega=\sqrt{-\frac{2 E}{M}}$.

Substituting (57) into (56), we obtain

$$
\begin{aligned}
G\left(\vec{\rho}, \overrightarrow{\rho^{\prime}} ; E\right) & =\frac{M \omega}{2 i \pi \hbar} \int_{0}^{\infty} \frac{d \sigma}{\sin (\omega \sigma)} \exp \left[\frac{i \sigma}{\hbar}\left(2 \alpha_{0}-\frac{\beta_{1}^{2}+\beta_{2}^{2}}{E}+i 0\right)\right] \\
& \times \exp \left\{\frac{i M \omega}{2 \hbar}\left[\left(\widetilde{\xi}^{2}+\widetilde{\eta}^{2}+\widetilde{\xi}^{\prime 2}+\eta^{\prime 2}\right) \cot (\omega \sigma)-\frac{2\left(\widetilde{\xi} \widetilde{\xi}^{\prime}+\widetilde{\eta} \widetilde{\eta}^{\prime}\right)}{\sin (\omega \sigma)}\right]\right\} .
\end{aligned}
$$

In order to determine the energy spectrum and the normalized wave functions of the bound states of the physical system, let's apply the Mehler formula ${ }^{39}$

$$
\frac{1}{\sqrt{1-a^{2}}} \exp \left\{-\frac{1}{2\left(1-a^{2}\right)}\left[\left(x^{2}+x^{2}\right)\left(1+a^{2}\right)-4 x x^{\prime} a\right]\right\}
$$




$$
=\exp \left[-\frac{1}{2}\left(x^{2}+x^{2}\right)\right] \sum_{n=0}^{\infty} \frac{1}{n !}\left(\frac{a}{2}\right)^{n} H_{n}(x) H_{n}\left(x^{\prime}\right) .
$$

With the help of an adequate change of variables, the poles of the Green's function (58) will be obtained thanks to an integration over $\sigma$; the discrete energy spectrum is found by solving the equation

$$
\omega^{3}-\frac{2 \alpha_{0}}{N \hbar} \omega^{2}-2 \frac{\beta_{1}^{2}+\beta_{2}^{2}}{N M \hbar}=0, \quad \text { or as well } \quad E_{N}=-\frac{M \omega_{N}^{2}}{2},
$$

with $N=n_{1}+n_{2}+1$. $\alpha_{0}$ being positive, then this cubic equation has one real root:

$$
\omega_{N}=\frac{2 \alpha_{0}}{3 N \hbar}+\lambda_{1}+\lambda_{2}
$$

where

$$
\lambda_{j}=\left[\left(\frac{2 \alpha_{0}}{3 N \hbar}\right)^{3}+\frac{\beta_{1}^{2}+\beta_{2}^{2}}{N M \hbar}+(-1)^{j} \sqrt{\frac{\beta_{1}^{2}+\beta_{2}^{2}}{N M \hbar}\left(\frac{\beta_{1}^{2}+\beta_{2}^{2}}{N M \hbar}+2\left(\frac{2 \alpha_{0}}{3 N \hbar}\right)^{3}\right.}\right]^{\frac{1}{3}} ;
$$

with $(j=1,2)$.

We may obtain the normalized wave functions of the bound states from the residues of the integrated expression of the Green's function (58),

$$
\begin{aligned}
\Psi_{n_{1}, n_{2}}(\xi, \eta) & =\frac{M}{\hbar}\left(\frac{1}{2^{N} n_{1} ! n_{2} ! N \pi} \lim _{\omega \rightarrow \omega_{N}} \frac{\omega^{3}\left(\omega^{2}-\omega_{N}^{2}\right)}{\omega^{3}-\frac{2 \alpha_{0}}{N \hbar} \omega^{2}-2 \frac{\beta_{1}^{2}+\beta_{2}^{2}}{N M \hbar}}\right)^{\frac{1}{2}} \\
\times & \exp \left[-\frac{M \omega_{N}}{2 \hbar}\left(\widetilde{\xi}^{2}+\widetilde{\eta}^{2}\right)\right] H_{n_{1}}\left(\sqrt{\frac{M \omega_{N}}{\hbar} \widetilde{\xi}}\right) H_{n_{2}}\left(\sqrt{\frac{M \omega_{N}}{\hbar}} \widetilde{\eta}\right) .
\end{aligned}
$$

Here, it is obvious that only states with an even total number of oscillator quanta contribute.

To find the wave functions of the continuous states, let's go back to the Green's function (58) and make use of the following relation

$$
\frac{1}{\sqrt{2 \pi \sin \alpha}} \exp (-(x+y) \cot \alpha) \exp \left(\frac{2 \sqrt{x y}}{\sin \alpha}\right)
$$




$$
\begin{aligned}
=\frac{1}{(2 \pi)^{2}} & \int_{-\infty}^{\infty} d p e^{(\pi-2 \alpha) p} \\
\times & {\left[\left|\Gamma\left(\frac{1}{4}+i p\right)\right|^{2} E_{-\frac{1}{2}+2 i p}^{(0)}\left(e^{-i \pi / 4} 2 \sqrt{x}\right) E_{-\frac{1}{2}-2 i p}^{(0)}\left(e^{i \pi / 4} 2 \sqrt{y}\right)\right.} \\
+ & \left.\left|\Gamma\left(\frac{3}{4}+i p\right)\right|^{2} E_{-\frac{1}{2}+2 i p}^{(1)}\left(e^{-i \pi / 4} 2 \sqrt{x}\right) E_{-\frac{1}{2}-2 i p}^{(1)}\left(e^{i \pi / 4} 2 \sqrt{y}\right)\right],
\end{aligned}
$$

which is established from the dispersion formula (28). The $E_{\nu}^{(0)}(z)$ and $E_{\nu}^{(1)}(z)$ are even and odd parabolic cylinder functions with respect to the variable $z$, respectively ${ }^{40}$. The poles of the continuous state Green's function will be obtained by integration on the $\tau$ variable. They will be given by

$$
\omega\left(p_{\xi}+p_{\eta}\right)-\frac{i}{\hbar}\left(\alpha_{0}-\frac{\beta_{1}^{2}+\beta_{2}^{2}}{E}\right)=0 .
$$

Then, by performing the change of variables $\left(p_{\xi}, p_{\eta}\right) \rightarrow\left[\frac{1}{2 p}\left(\frac{1}{\tilde{a}}+\varsigma\right), \frac{1}{2 p}\left(\frac{1}{\tilde{a}}-\varsigma\right)\right]$ where $\widetilde{a}=\frac{\hbar^{2}}{M\left(\alpha_{0}-M\left(\beta_{1}^{2}+\beta_{2}^{2}\right) / \hbar^{2} p^{2}\right)}$, it is possible to write the contribution of the continuous part to the Green's function as

$$
G^{c}\left(\xi, \eta, \xi^{\prime}, \eta^{\prime} ; E\right)=i \hbar \int_{-\infty}^{\infty} d p \int_{-\infty}^{\infty} d \varsigma \frac{\Psi_{p, \varsigma}^{*}\left(\xi^{\prime}, \eta^{\prime}\right) \Psi_{p, \varsigma}(\xi, \eta)}{E+i 0-E_{p}}
$$

where $E_{p}=-\frac{M \omega^{2}}{2}=\frac{\hbar^{2} p^{2}}{2 M}$, and the continuous functions $\Psi_{p, \varsigma}(\xi, \eta)$ have the form

$$
\begin{aligned}
\Psi_{p, \varsigma}(\xi, \eta)= & \frac{e^{\pi / 2 \widetilde{a} p}}{4 \pi \sqrt{2}}\left(\begin{array}{c}
\Gamma\left(\frac{1}{4}+\frac{i}{2 p}\left(\frac{1}{\tilde{a}}+\varsigma\right)\right) E_{-\frac{1}{2}+\frac{i}{p}\left(\frac{1}{\tilde{a}}+\varsigma\right)}^{(0)}\left(e^{-i \pi / 4} \sqrt{2 p} \widetilde{\xi}\right) \\
\Gamma\left(\frac{3}{4}+\frac{i}{2 p}\left(\frac{1}{\tilde{a}}+\varsigma\right)\right) E_{-\frac{1}{2}+\frac{i}{p}\left(\frac{1}{\tilde{a}}+\varsigma\right)}^{(1)}\left(e^{-i \pi / 4} \sqrt{2 p} \widetilde{\xi}\right)
\end{array}\right) \\
& \times\left(\begin{array}{c}
\Gamma\left(\frac{1}{4}+\frac{i}{2 p}\left(\frac{1}{\tilde{a}}-\varsigma\right)\right) E_{-\frac{1}{2}+\frac{i}{p}\left(\frac{1}{\tilde{a}}-\varsigma\right)}^{(0)}\left(e^{-i \pi / 4} \sqrt{2 p} \widetilde{\eta}\right) \\
\Gamma\left(\frac{3}{4}+\frac{i}{2 p}\left(\frac{1}{\tilde{a}}-\varsigma\right)\right) E_{-\frac{1}{2}+\frac{i}{p}\left(\frac{1}{\tilde{a}}-\varsigma\right)}^{(1)}\left(e^{-i \pi / 4} \sqrt{2 p} \widetilde{\eta}\right)
\end{array}\right) .
\end{aligned}
$$




\section{Three-dimensional maximally super-integrable po- tentials}

In three-dimensional Euclidean space, Smorodinsky and co-workers have found a set of five potentials which have five functionally independent integrals of motion. These three-dimensional potentials are called maximally super-integrable potentials. At least, each potential of the so-called class of Smorodinsky-Winternitz potentials can be treated in two coordinate systems through the so $(2,1)$ Lie algebraic approach. Here, we shall restrict ourselves to study the potential

$$
V_{3}(\vec{r})=-\frac{\alpha_{0}}{r}+\frac{\hbar^{2}}{2 M}\left(\frac{k_{1}^{2}-\frac{1}{4}}{x_{1}^{2}}+\frac{k_{2}^{2}-\frac{1}{4}}{x_{2}^{2}}\right) .
$$

The integrals of motion are

$$
\left\{\begin{array}{l}
H_{3}=\frac{p^{2}}{2 M}+V_{3}(\vec{r}), \quad I_{1}=\frac{L_{3}^{2}}{2 M}+\frac{\hbar^{2}}{2 M}\left(\frac{k_{1}^{2}-\frac{1}{4}}{\cos ^{2} \phi}+\frac{k_{2}^{2}-\frac{1}{4}}{\sin ^{2} \phi}\right), \\
I_{2}=\frac{L_{2}^{2}}{2 M}+\frac{\hbar^{2}}{2 M} \frac{k_{1}^{2}-\frac{1}{4}}{\tan ^{2} \theta \cos ^{2} \phi}, \quad I_{3}=\frac{\vec{L}^{2}}{2 M}+\frac{\hbar^{2}}{2 M \sin ^{2} \theta}\left(\frac{k_{1}^{2}-\frac{1}{4}}{\cos ^{2} \phi}+\frac{k_{2}^{2}-\frac{1}{4}}{\sin ^{2} \phi}\right), \\
I_{4}=\frac{1}{4 M}\left(I_{x_{1} x_{2}}+I_{x_{2} x_{1}}\right)-(\xi-\eta)\left[\frac{\alpha}{\xi+\eta}-\frac{\hbar^{2}}{2 M \xi \eta}\left(\frac{k_{1}^{2}-\frac{1}{4}}{\cos ^{2} \phi}+\frac{k_{2}^{2}-\frac{1}{4}}{\sin ^{2} \phi}\right)\right],
\end{array}\right.
$$

where $I_{i j}=\left\{L_{i}, P_{j}\right\}=L_{i} P_{j}+P_{j} L_{i}$ with $(i, j) \equiv\left(x_{1}, x_{2}, x_{3}\right)$.

This potential is a generalization of the Coulomb potential analyzed by various authors in the path integral ${ }^{41-52}$ and algebraic approach ${ }^{53,54}$ contexts. As it features singularities for $x_{1}=0$ and $x_{2}=0$, all we need is to discuss it in the $0<x_{1}, x_{2}<\infty$ and $x_{3} \in R$ area. It is possible to evaluate the Green's function

$$
G\left(\vec{r}, \overrightarrow{r^{\prime}} ; E\right)=\int_{0}^{\infty} d S \exp \left[-\frac{i S}{\hbar}\left(H_{3}-E-i 0\right)\right] \delta\left(\vec{r}-\overrightarrow{r^{\prime}}\right)
$$

in parabolic and spherical coordinates.

\section{Parabolic coordinates}

For given parabolic coordinates $x_{1}=\xi \eta \cos \phi, x_{2}=\xi \eta \sin \phi$, $x_{3}=\frac{1}{2}\left(\xi^{2}-\eta^{2}\right), \xi, \eta>0$ and $0 \leq \phi<2 \pi$, the Green's function (70) can be written

$$
G(\vec{r}, \vec{r} ; E)=\int_{0}^{\infty} d S \exp \left[-\frac{i S}{\hbar}\left(\widetilde{H}_{3}-E-i 0\right)\right] \frac{\delta\left(\xi-\xi^{\prime}\right) \delta\left(\eta-\eta^{\prime}\right) \delta\left(\phi-\phi^{\prime}\right)}{\xi \eta\left(\xi^{2}+\eta^{2}\right)},
$$


with

$$
\begin{aligned}
\widetilde{H}_{3}= & -\frac{\hbar^{2}}{2 M}\left[\frac{1}{\xi^{2}+\eta^{2}}\left(\frac{\partial^{2}}{\partial \xi^{2}}+\frac{1}{\xi} \frac{\partial}{\partial \xi}+\frac{\partial^{2}}{\partial \eta^{2}}+\frac{1}{\eta} \frac{\partial}{\partial \eta}\right)+\frac{1}{\xi^{2} \eta^{2}} \frac{\partial^{2}}{\partial \phi^{2}}\right] \\
& -\frac{2 \alpha_{0}}{\xi^{2}+\eta^{2}}+\frac{\hbar^{2}}{2 M} \frac{1}{\xi^{2} \eta^{2}}\left(\frac{k_{1}^{2}-\frac{1}{4}}{\cos ^{2} \phi}+\frac{k_{2}^{2}-\frac{1}{4}}{\sin ^{2} \phi}\right) .
\end{aligned}
$$

Separating the angular part of the expression (71) by a time transformation defined by $\tau=\frac{S}{\xi^{2} \eta^{2}}$, we can deduce that

$$
G\left(\vec{r}, \overrightarrow{r^{\prime}}, E\right)=\int_{0}^{\infty} d \tau K\left(\xi, \eta, \xi^{\prime}, \eta^{\prime} ; \tau\right) K\left(\phi, \phi^{\prime} ; \tau\right)
$$

where

$$
\begin{aligned}
K\left(\xi, \eta, \xi^{\prime}, \eta^{\prime} ; \tau\right)= & \exp \left\{-\frac{i \tau}{\hbar}\left[H_{3}(\xi, \eta)-E \xi^{2} \eta^{2}-i 0\right]\right\} \\
& \times \frac{\xi \eta}{\xi^{2}+\eta^{2}} \delta\left(\xi-\xi^{\prime}\right) \delta\left(\eta-\eta^{\prime}\right)
\end{aligned}
$$

and

$$
\begin{aligned}
K\left(\phi, \phi^{\prime} ; \tau\right) & =\exp \left\{-\frac{i \tau}{\hbar} \frac{\hbar^{2}}{2 M}\left(-\frac{\partial^{2}}{\partial \phi^{2}}+\frac{k_{1}^{2}-\frac{1}{4}}{\cos ^{2} \phi}+\frac{k_{2}^{2}-\frac{1}{4}}{\sin ^{2} \phi}\right)\right\} \delta\left(\phi-\phi^{\prime}\right) \\
& =\sum_{n=0}^{\infty} \Phi_{n}^{\left( \pm k_{2}, \pm k_{1}\right)}(\phi) \Phi_{n}^{\left( \pm k_{2}, \pm k_{1}\right)}\left(\phi^{\prime}\right) \exp \left(-\frac{i}{\hbar} \frac{\hbar^{2} \lambda_{1}^{2}}{2 M} \tau\right)
\end{aligned}
$$

with $\lambda_{1}=2 n \pm k_{1} \pm k_{2}+1$.

It is to be noted that the explicit construction of the kernel $K\left(\phi, \phi^{\prime} ; \tau\right)$ thanks the (MS) variant of the algebraic technique is being investigated and will be the subject of our forthcoming publication.

Introducing (75) into (73) and applying the $\tau \rightarrow S$ inverse time transformation will allow us to write (73) as follows:

$$
\begin{aligned}
G\left(\vec{r}, \overrightarrow{r^{\prime}} ; E\right)= & \sum_{n=0}^{\infty} \Phi_{n}^{\left( \pm k_{2}, \pm k_{1}\right)}(\phi) \Phi_{n}^{\left( \pm k_{2}, \pm k_{1}\right)}\left(\phi^{\prime}\right) \int_{0}^{\infty} d S \exp \left[\frac{i S}{\hbar}(E-i 0)\right] \\
& \times \exp \left\{-\frac{i S}{\hbar}\left[\widetilde{H}_{3}(\xi, \eta)\right]\right\} \frac{\delta\left(\xi-\xi^{\prime}\right) \delta\left(\eta-\eta^{\prime}\right)}{\xi \eta\left(\xi^{2}+\eta^{2}\right)}
\end{aligned}
$$


where

$$
\widetilde{H}_{3}(\xi, \eta)=-\frac{\hbar^{2}}{2 M} \frac{1}{\xi^{2}+\eta^{2}}\left(\frac{\partial^{2}}{\partial \xi^{2}}+\frac{1}{\xi} \frac{\partial}{\partial \xi}+\frac{\partial^{2}}{\partial \eta^{2}}+\frac{1}{\eta} \frac{\partial}{\partial \eta}\right)-\frac{2 \alpha_{0}}{\xi^{2}+\eta^{2}}+\frac{\hbar^{2} \lambda_{1}^{2}}{2 M \xi^{2} \eta^{2}} .
$$

If we now eliminate the $\frac{1}{\xi} \frac{\partial}{\partial \xi}$ and $\frac{1}{\eta} \frac{\partial}{\partial \eta}$ operators by applying $\frac{\partial^{2}}{\partial \xi^{2}}+\frac{1}{\xi} \frac{\partial}{\partial \xi}$ and $\frac{\partial^{2}}{\partial \eta^{2}}+\frac{1}{\eta} \frac{\partial}{\partial \eta}$ on $\frac{\delta\left(\xi-\xi^{\prime}\right)}{\sqrt{\xi \xi^{\prime}}}$ and $\frac{\delta\left(\eta-\eta^{\prime}\right)}{\sqrt{\eta \eta^{\prime}}}$ respectively, we can then proceed with a new $S \rightarrow \sigma$ time transformation defined by $\sigma=\frac{S}{\xi^{2}+\eta^{2}}$, which will allow us to write

$$
\begin{aligned}
G\left(\vec{r}, \overrightarrow{r^{\prime}} ; E\right)= & \sum_{n=0}^{\infty} \Phi_{n}^{\left( \pm k_{2}, \pm k_{1}\right)}(\phi) \Phi_{n}^{\left( \pm k_{2}, \pm k_{1}\right)}\left(\phi^{\prime}\right) \int_{0}^{\infty} d \sigma \exp \left[\frac{i}{\hbar}\left(2 \alpha_{0}+i 0\right) \sigma\right] \\
& \times K_{n}\left(\xi, \xi^{\prime} ; \sigma\right) K_{n}\left(\eta, \eta^{\prime} ; \sigma\right)
\end{aligned}
$$

with

$$
K_{n}\left(u, u^{\prime} ; \sigma\right)=\frac{1}{\sqrt{u u^{\prime}}} \exp \left\{-\frac{i \sigma}{\hbar}\left[-\frac{\hbar^{2}}{2 M}\left(\frac{\partial^{2}}{\partial u^{2}}-\frac{\lambda_{1}^{2}-\frac{1}{4}}{u^{2}}\right)-E u^{2}\right]\right\} \delta\left(u-u^{\prime}\right),
$$

where $u \equiv(\xi$ or $\eta)$.

So, it is possible to give the kernel (79) in function of the so(2,1) Lie algebra operators. Indeed, by following the equations (2) and (13), we obtain

$$
\begin{aligned}
K_{n}\left(u, u^{\prime} ; \sigma\right) & =\frac{1}{\sqrt{u u^{\prime}}} \exp \left\{-\frac{i \sigma}{\hbar}\left[T_{1}(u)+2 \hbar^{2} \omega^{2} T_{3}(u)\right]\right\} \delta\left(u-u^{\prime}\right) \\
& =\frac{M \omega}{i \hbar \sin (\omega \sigma)} \exp \left[\frac{i M \omega}{2 \hbar}\left(u^{2}+u^{\prime 2}\right) \cot (\omega \sigma)\right] I_{\lambda_{1}}\left(\frac{M \omega u u^{\prime}}{i \hbar \sin (\omega \sigma)}\right),
\end{aligned}
$$

with $\omega=\sqrt{-\frac{2 E}{M}}$. The Green's function (78) can now be written

$$
G\left(\vec{r}, \overrightarrow{r^{\prime}} ; E\right)=\sum_{n=0}^{\infty} \Phi_{n}^{\left( \pm k_{2}, \pm k_{1}\right)}(\phi) \Phi_{n}^{\left( \pm k_{2}, \pm k_{1}\right)}\left(\phi^{\prime}\right) G_{n}\left(\xi, \eta, \xi^{\prime}, \eta^{\prime} ; E\right),
$$

with

$$
\begin{aligned}
G_{n}\left(\xi, \eta, \xi^{\prime}, \eta^{\prime} ; E\right)= & \left(\frac{M \omega}{i \hbar}\right)^{2} \int_{0}^{\infty} \frac{d \sigma}{\sin ^{2}(\omega \sigma)} \exp \left[\frac{i}{\hbar}\left(2 \alpha_{0}+i 0\right) \sigma\right] \\
& \times \exp \left[\frac{i M \omega}{2 \hbar}\left(\xi^{2}+\eta^{2}+\xi^{\prime 2}+\eta^{\prime 2}\right) \cot (\omega \sigma)\right] \\
& \times I_{\lambda_{1}}\left(\frac{M \omega \xi \xi^{\prime}}{i \hbar \sin (\omega \sigma)}\right) I_{\lambda_{1}}\left(\frac{M \omega \eta \eta^{\prime}}{i \hbar \sin (\omega \sigma)}\right) .
\end{aligned}
$$


Thanks to the Hille and Hardy formula (23) for the discrete part and thanks to the scattering relation (28) for the continuous part, the Green's function (81) can be developed into partial waves as follows:

$$
\begin{aligned}
G\left(\vec{r}, \overrightarrow{r^{\prime}} ; E\right)= & i \hbar \sum_{n=0}^{\infty}\left\{\sum_{n_{1}, n_{2}=0}^{\infty} \frac{\Psi_{n_{1} n_{2}, n}(\xi, \eta, \phi) \Psi_{n_{1}, n_{2}, n}^{*}\left(\xi^{\prime}, \eta^{\prime}, \phi^{\prime}\right)}{E+i 0-E_{N}}\right. \\
& \left.+\int_{0}^{\infty} d p \int_{-\infty}^{\infty} d \kappa \frac{\Psi_{p, \kappa, n}(\xi, \eta, \phi) \Psi_{p, \kappa, n}^{*}\left(\xi^{\prime}, \eta^{\prime}, \phi^{\prime}\right)}{E+i 0-E_{p}}\right\}
\end{aligned}
$$

Hence, for bound states-the normalized wave functions and energy spectrum will be

$$
\begin{aligned}
& \Psi_{n_{1}, n_{2}, n}(\xi, \eta, \phi)= {\left[\frac{2}{a^{3} N^{4}} \frac{n_{1} ! n_{2} !}{\Gamma\left(n_{1}+\lambda_{1}+1\right) \Gamma\left(n_{2}+\lambda_{2}+1\right)}\right]^{\frac{1}{2}}\left(\frac{\xi \eta}{a N}\right)^{\lambda_{1}} } \\
& \times \exp \left(-\frac{\xi^{2}+\eta^{2}}{2 a N}\right) L_{n_{1}}^{\lambda_{1}}\left(\frac{\xi^{2}}{a N}\right) L_{n_{2}}^{\lambda_{1}}\left(\frac{\eta^{2}}{a N}\right) \Phi_{n}^{\left( \pm k_{2}, \pm k_{1}\right)}(\phi), \\
& E_{N}=-\frac{M \alpha_{0}^{2}}{2 \hbar^{2} N^{2}}, \quad N=n_{1}+n_{2}+\lambda_{1}+1,
\end{aligned}
$$

and, for the continuous states, the normalized wave functions and the energy spectrum will be

$$
\begin{gathered}
\Psi_{p, \kappa, n}(\xi, \eta, \phi)=\frac{\left|\Gamma\left(\frac{1}{2}+\frac{\lambda_{1}}{2}+\frac{i}{2 p}\left(\frac{1}{a}+\kappa\right)\right) \Gamma\left(\frac{1}{2}+\frac{\lambda_{1}}{2}+\frac{i}{2 p}\left(\frac{1}{a}-\kappa\right)\right)\right|}{2 \pi \Gamma^{2}\left(\lambda_{1}+1\right) \xi \eta} \\
\times \frac{e^{\frac{\pi}{2 a p}}}{\sqrt{p}} M_{-\frac{i}{2 p}\left(\frac{1}{a}+\kappa\right), \frac{\lambda_{1}}{2}}\left(-i p \xi^{2}\right) M_{-\frac{i}{2 p}\left(\frac{1}{a}-\kappa\right), \frac{\lambda_{1}}{2}}\left(-i p \eta^{2}\right) \\
\Phi_{n}^{\left( \pm k_{2}, \pm k_{1}\right)}(\phi), \\
E_{p}=\frac{\hbar^{2} p^{2}}{2 M} .
\end{gathered}
$$

\section{Spherical coordinates}

To study the problem in this coordinate system, we shall use the expression of the partial Green's function (82) and use the following change of 
variables

$$
(\xi, \eta) \rightarrow\left(\sqrt{2 r} \cos \frac{\theta}{2}, \sqrt{2 r} \sin \frac{\theta}{2}\right) .
$$

The partial Green's function (82) then can be written

$$
\begin{aligned}
G_{n}\left(r, \theta, r^{\prime}, \theta^{\prime} ; E\right)= & \sum_{m=0}^{\infty}\left(m+\lambda_{1}+\frac{1}{2}\right) \frac{\Gamma\left(m+2 \lambda_{1}+1\right)}{m !} P_{m+\lambda_{1}}^{-\lambda_{1}}(\cos \theta) \\
& \times P_{m+\lambda_{1}}^{-\lambda_{1}}\left(\cos \theta^{\prime}\right) \frac{2 M \omega}{i \hbar \sqrt{r r^{\prime}}} \int_{0}^{\infty} \frac{d \sigma}{\sin (\omega \sigma)} \exp \left[\frac{i}{\hbar}(2 \alpha+i 0) \sigma\right] \\
& \times \exp \left[\frac{i M \omega}{\hbar}\left(r+r^{\prime}\right) \cot (\omega \sigma)\right] I_{2 m+2 \lambda_{1}+1}\left(\frac{2 M \omega \sqrt{r r^{\prime}}}{i \hbar \sin (\omega \sigma)}\right) .
\end{aligned}
$$

We have used here the addition theorem (36), the connection between hypergeometric functions and the Jacobi polynomials (see Ref. ${ }^{37}$, p. 1036, Eq. $(8.962))$

$$
{ }_{2} F_{1}\left(l+\alpha+\beta+1,-l ; 1+\alpha ; \frac{1-t}{2}\right)=\frac{l ! \Gamma(\alpha+1)}{\Gamma(l+\alpha+1)} P_{l}^{(\alpha, \beta)}(t),
$$

as well as the relation between the hypergeometric functions and the Legendre polynomials (see Ref. ${ }^{37}$, p. 1009, Eq. (8.771)), and eventually the link between $P_{\nu}^{-m}(x)$ and $P_{\nu}^{m}(x)$ (see Ref. ${ }^{37}$, p. 1008, Eq. (8.752.2)).

In order to perform the integration on the $\sigma$ time variable, we shall use the formula (40). Consequently, the final expression of the Green's function in spherical coordinates will be

$$
\begin{aligned}
G\left(\vec{r}, \overrightarrow{r^{\prime}} ; E\right)= & \sum_{n=0}^{\infty} \sum_{m=0}^{\infty}\left(m+\lambda_{1}+\frac{1}{2}\right) \frac{\Gamma\left(m+\lambda_{1}+1\right)}{m !} \frac{\Gamma\left(p+m+\lambda_{1}+1\right)}{\Gamma\left(2 m+2 \lambda_{1}+2\right)} \\
& \times \frac{1}{i \omega r r^{\prime}} M_{-p, m+\lambda_{1}+\frac{1}{2}}\left(\frac{2 M \omega}{\hbar} r^{\prime}\right) W_{-p, m+\lambda_{1}+\frac{1}{2}}\left(\frac{2 M \omega}{\hbar} r\right) \\
& \times P_{m+\lambda_{1}}^{-\lambda_{1}}(\cos \theta) P_{m+\lambda_{1}}^{-\lambda_{1}}\left(\cos \theta^{\prime}\right) \Phi_{n}^{\left( \pm k_{2}, \pm k_{1}\right)}(\phi) \Phi_{n}^{\left( \pm k_{2}, \pm k_{1}\right)}\left(\phi^{\prime}\right),
\end{aligned}
$$

where $r>r^{\prime}$ and $p=-\frac{\alpha_{0}}{\hbar \omega}$. 
The Hille and Hardy formula (23) and an analytic proceeding consisting in using the Sommerfeld-Watson transformation ${ }^{55}$ will help us to write the Green's function (91) in the form of a partial wave development consisting of two contributions of a discrete and a continuous part:

$$
\begin{aligned}
G\left(\vec{r}, \overrightarrow{r^{\prime}} ; E\right)= & i \hbar \sum_{n, m=0}^{\infty}\left\{\sum_{l=0}^{\infty} \frac{\Psi_{l, m, n}(r, \theta, \phi) \Psi_{l, m, n}^{*}\left(r^{\prime}, \theta^{\prime}, \phi^{\prime}\right)}{E+i 0-E_{N}}\right. \\
& \left.+\int_{0}^{\infty} d k \frac{\Psi_{k, m, n}(r, \theta, \phi) \Psi_{k, m, n}^{*}\left(r^{\prime}, \theta^{\prime}, \phi^{\prime}\right)}{E+i 0-\frac{\hbar^{2} k^{2}}{2 M}}\right\},
\end{aligned}
$$

with-for bound states-the poles located around the values of $E$ and the normalized wave functions respectively given by

$$
\begin{aligned}
E_{N} & =-\frac{M \alpha_{0}^{2}}{2 \hbar^{2} N^{2}} ; \quad N=l+m+\lambda_{1}+1 \\
\Psi_{l, m, n}(r, \theta, \phi)= & {\left[\frac{\left(m+\lambda_{1}+\frac{1}{2}\right) l ! \Gamma\left(m+\lambda_{1}+1\right)}{a^{3}\left(l+\lambda_{2}+\frac{1}{2}\right)^{4} m ! \Gamma\left(l+2 \lambda_{2}+1\right)}\right]^{\frac{1}{2}}\left(\frac{2 r}{a\left(l+\lambda_{2}+\frac{1}{2}\right)}\right)^{\lambda_{2}-\frac{1}{2}} } \\
& \times \exp \left(-\frac{r}{a\left(l+\lambda_{2}+\frac{1}{2}\right)}\right) L_{l}^{2 \lambda_{2}}\left(\frac{2 r}{a\left(l+\lambda_{2}+\frac{1}{2}\right)}\right) \\
& \times P_{m+\lambda_{1}}^{-\lambda_{1}}(\cos \theta) \Phi_{n}^{\left( \pm k_{2}, \pm k_{1}\right)}(\phi) .
\end{aligned}
$$

For continuous states, the normalized wave functions and energy spectrum are respectively given by

$$
\begin{aligned}
\Psi_{k, m, n}(r, \theta, \phi)= & \frac{1}{\sqrt{2 \pi}}\left[\left(m+\lambda_{1}+\frac{1}{2}\right) \frac{\Gamma\left(m+\lambda_{1}+1\right)}{m !}\right]^{\frac{1}{2}} \frac{\left|\Gamma\left(\frac{1}{2}+\lambda_{2}-\frac{i}{a k}\right)\right|}{\Gamma\left(2 \lambda_{2}+1\right)} \\
& \times \frac{e^{\frac{\pi}{2 a k}}}{r} M_{\frac{i}{a k}, \lambda_{2}}(-2 i k r) P_{m+\lambda_{1}}^{-\lambda_{1}}(\cos \theta) \Phi_{n}^{\left( \pm k_{2}, \pm k_{1}\right)}(\phi),
\end{aligned}
$$

\section{Three-dimensional minimally super-integrable po- tentials}

There are nine three-dimensional potentials which belong to the class

of minimally super-integrable Smorodinsky-Winternitz potentials, that is to 
say three-dimensional potentials characterized by the existence of four functionally independent integrals of motion. Among them are seven potentials which have $\mathrm{SO}(2,1)$ as a dynamical group and thus their exact solution can be given via the (M-S) variant of the algebraic approach in different coordinate systems. As an example, we shall discuss the potential

$$
V_{4}(\vec{r})=-\frac{\alpha_{0}}{r}+\frac{\hbar^{2}}{2 M\left(x_{1}^{2}+x_{2}^{2}\right)}\left(\frac{k_{1}^{2} x_{3}}{r}+F\left(\frac{x_{2}}{x_{1}}\right)\right),
$$

with $k_{1}$ a positive constant. The corresponding observables have the form

$$
\left\{\begin{array}{c}
H_{4}=\frac{\vec{P}^{2}}{2 M}+V_{4}(\vec{r}), I_{1}=\frac{L_{z}^{2}}{2 M}+F(\tan \phi), \\
I_{2}=\frac{P_{z}^{2}}{2 M}+\frac{\hbar^{2}}{2 M} \frac{k_{1}^{2} \cos \theta+F(\tan \phi)}{\sin ^{2} \theta}, \\
I_{3}=\frac{1}{4 M}\left(I_{x_{1} x_{2}}+I_{x_{2} x_{1}}\right)-\alpha_{0} \frac{\xi-\eta}{\xi+\eta}+\frac{\hbar^{2}}{2 M}\left(\frac{1}{\eta}-\frac{1}{\xi}\right)\left(k_{1}^{2}+F(\tan \phi)\right),
\end{array}\right.
$$

where $I_{i j}=\left\{L_{i}, P_{j}\right\}=L_{i} P_{j}+P_{j} L_{i}$ with $(i, j) \equiv\left(x_{1}, x_{2}, x_{3}\right)$

The Green's function for this potential can be explicitly evaluated in the parabolic and spherical coordinate systems. For $F\left(\frac{y}{x}\right)=\gamma^{2}$ and $\gamma$ a real constant, this potential reduces to the ring-shaped potential proposed by Hartmann as a model for the ring-shaped molecules. It has been analyzed by many authors in the framework of path integrals ${ }^{56-60}$ and through the algebraic approach ${ }^{61-68}$.We can also notice the close link of the latter with the Coulomb potential plus the barrier created by the solenoid of AharonovBohm $^{69}$ treated with the path integrals ${ }^{70-72}$ and via the algebraic technique ${ }^{73}$ . To give the solution for the potential (96) via the (MS) variant of the so(2,1) algebraic approach, we will use the Kustaanheimo-Stiefel transformation ${ }^{74}$ $\left\{x_{1}, x_{2}, x_{3}\right\} \rightarrow\left\{u_{j, j} \in[1,4]\right\}$ corresponding to the surjection $R^{4} \rightarrow R^{3}$, which can be defined as:

$$
\left(\begin{array}{l}
x_{1} \\
x_{2} \\
x_{3}
\end{array}\right)=(A)\left(\begin{array}{l}
u_{1} \\
u_{2} \\
u_{3} \\
u_{4}
\end{array}\right), \quad(A)=\left(\begin{array}{cccc}
u_{3} & u_{4} & u_{1} & u_{2} \\
-u_{4} & u_{3} & u_{2} & -u_{1} \\
-u_{1} & -u_{2} & u_{3} & u_{4} \\
-u_{2} & u_{1} & -u_{4} & u_{3}
\end{array}\right)
$$

with the constraint

$$
d x_{4}=2\left(-u_{2} d u_{1}+u_{1} d u_{2}-u_{4} d u_{3}+u_{3} d u_{4}\right)=0
$$


allowing to define a fourth coordinate

$$
x_{4}=2 \int^{s}\left(-u_{2} \dot{u}_{1}+u_{1} \dot{u}_{2}-u_{4} \dot{u}_{3}+u_{3} \dot{u}_{4}\right) d s .
$$

Moreover, we can show that

$$
\left(\begin{array}{c}
\frac{\partial}{\partial x_{1}} \\
\frac{\partial}{\partial x_{2}} \\
\frac{\partial}{\partial x_{3}} \\
\frac{1}{2 r} \frac{\partial}{\partial x_{4}}
\end{array}\right)=\frac{1}{2 r}(A)\left(\begin{array}{c}
\frac{\partial}{\partial u_{1}} \\
\frac{\partial}{\partial u_{2}} \\
\frac{\partial}{\partial u_{3}} \\
\frac{\partial}{\partial u_{4}}
\end{array}\right),
$$

The (KS) transformation allows us to write the Laplacian $\vec{\nabla}^{2}$ in $R^{3}$ in terms of the laplacian $\square^{2}$ in $R^{4}$ as

$$
\vec{\nabla}^{2}=\frac{1}{4 r} \square^{2}-\frac{1}{4 r^{2}} \frac{\partial^{2}}{\partial x_{4}^{2}}, \quad \square^{2}=\sum_{j=1}^{4} \frac{\partial^{2}}{\partial u_{j}^{2}}
$$

where $r=\left(x_{1}^{2}+x_{2}^{2}+x_{3}^{2}\right)^{\frac{1}{2}}=u_{1}^{2}+u_{2}^{2}+u_{3}^{2}+u_{4}^{2}$.

Using the Schwinger's integral representation, the Green's function relative to the potential $V_{4}(\vec{r})$ is written

$$
G(\vec{r}, \vec{r} ; E)=\int_{0}^{\infty} d S \exp \left\{-\frac{i S}{\hbar}\left[-\frac{\hbar^{2}}{2 M} \vec{\nabla}^{2}+V_{4}(\vec{r})-E-i 0\right]\right\} \delta\left(\vec{r}-\overrightarrow{r^{\prime}}\right) .
$$

It is possible to introduce an additional variable $x_{4}$ by means of the wellknown identity

$$
\int_{-\infty}^{\infty} \exp \left[\frac{i}{\hbar}\left(\frac{\hbar^{2} S}{8 M r^{2}} \frac{\partial^{2}}{\partial x_{4}^{2}}\right)\right] \delta\left(x_{4}\right) d x_{4}=1
$$

and to show that expression (104) can be written as

$$
G\left(\vec{r}, \overrightarrow{r^{\prime}} ; E\right)=\int_{-\infty}^{\infty} d x_{4} G\left(\vec{r}, x_{4}, \overrightarrow{r^{\prime}}, 0 ; E\right)
$$

where

$$
G\left(\vec{r}, x_{4}, \overrightarrow{r^{\prime}}, 0 ; E\right)=\int_{0}^{\infty} d S \exp \left\{-\frac{i S}{\hbar}\left[H_{T}-E-i 0\right]\right\} \delta\left(\vec{r}-\overrightarrow{r^{\prime}}\right) \delta\left(x_{4}\right),
$$


with

$$
H_{T}=-\frac{\hbar^{2}}{2 M}\left(\vec{\nabla}^{2}+\frac{1}{2 r^{2}} \frac{\partial^{2}}{\partial x_{4}^{2}}\right)+V_{4}(\vec{r}) .
$$

Using (102), (98), the Jacobian of this transformation given by $\frac{\partial\left(x_{1}, x_{2}, x_{3}, x_{4}\right)}{\partial\left(u_{1}, u_{2}, u_{3}, u_{4}\right)}=16 r^{2}$, and the time transformation $\tau=\frac{S}{4 r}$, the Green's function (106 ) can be put in the form

$$
\begin{aligned}
G\left(\vec{r}, x_{4}, \overrightarrow{r^{\prime}}, 0 ; E\right)= & \frac{1}{4 r} \int_{0}^{\infty} d \tau \exp \left[\frac{i}{\hbar}\left(4 \alpha_{0}+i 0\right) \tau\right] \\
& \times \exp \left\{-\frac{i \tau}{\hbar}\left[-\frac{\hbar^{2}}{2 M} \square^{2}+V(u)\right]\right\} \prod_{j=1}^{4} \delta\left(u_{j}-u_{j}^{\prime}\right),
\end{aligned}
$$

where

$$
\begin{aligned}
V(u)= & \frac{\hbar^{2} k_{1}^{2}}{2 M}\left(\frac{1}{u_{1}^{2}+u_{2}^{2}}-\frac{1}{u_{3}^{2}+u_{4}^{2}}\right)+\frac{\hbar^{2}}{2 M}\left(\frac{1}{u_{1}^{2}+u_{2}^{2}}\right. \\
& \left.+\frac{1}{u_{3}^{2}+u_{4}^{2}}\right) F\left(\frac{u_{2} u_{3}-u_{1} u_{4}}{u_{1} u_{3}+u_{2} u_{4}}\right)-4 E r .
\end{aligned}
$$

The evaluation of this expression is possible in two coordinate systems.

\section{Parabolic coordinates}

Going on to the double polar coordinates

$$
\begin{cases}\left(u_{1}, u_{2}\right) \rightarrow\left(\eta, \phi_{1}\right): u_{1}=\frac{\eta}{\sqrt{2}} \cos \phi_{1}, & u_{2}=\frac{\eta}{\sqrt{2}} \sin \phi_{1} \\ \left(u_{3}, u_{4}\right) \rightarrow\left(\xi, \phi_{2}\right): u_{3}=\frac{\xi}{\sqrt{2}} \cos \phi_{2}, & u_{4}=\frac{\xi}{\sqrt{2}} \sin \phi_{2},\end{cases}
$$

the Green's function ( 108 ) becomes

$$
\begin{aligned}
G\left(\vec{r}, x_{4}, \vec{r}, 0 ; E\right)= & \frac{1}{2 r} \int_{0}^{\infty} d \tau \exp \left[\frac{i}{\hbar}\left(2 \alpha_{0}+i 0\right) \tau\right] \exp \left[-\frac{i \tau}{\hbar} H_{p}\right] \\
& \times \frac{\delta\left(\xi-\xi^{\prime}\right) \delta\left(\eta-\eta^{\prime}\right)}{\sqrt{\xi \xi^{\prime} \eta \eta^{\prime}}} \delta\left(\phi_{1}-\phi_{1}^{\prime}\right) \delta\left(\phi_{2}-\phi_{2}^{\prime}\right),
\end{aligned}
$$


where

$$
H_{p}=-\frac{\hbar^{2}}{2 M}\left(\frac{\partial^{2}}{\partial \xi^{2}}+\frac{\partial^{2}}{\partial \eta^{2}}+\frac{1}{\xi} \frac{\partial}{\partial \xi}+\frac{1}{\eta} \frac{\partial}{\partial \eta}+\frac{1}{\xi^{2}} \frac{\partial^{2}}{\partial \phi_{1}^{2}}+\frac{1}{\eta^{2}} \frac{\partial^{2}}{\partial \phi_{2}^{2}}\right)+V_{p},
$$

with

$$
V_{p}=-\frac{\hbar^{2} k_{1}^{2}}{2 M}\left(\frac{1}{\xi^{2}}-\frac{1}{\eta^{2}}\right)+\frac{\hbar^{2}}{2 M}\left(\frac{1}{\xi^{2}}+\frac{1}{\eta^{2}}\right) F\left(\tan \left(\phi_{1}-\phi_{2}\right)-E\left(\xi^{2}+\eta^{2}\right) .\right.
$$

Here we have applied the rescaling of $2 \tau$ to $\tau$.

At this point, we notice that the separation of variables is not possible. To achieve it, it is necessary to perform the integration on the variable $x_{4}$ by using the Euler's angles $\phi_{1}=\frac{\alpha+\phi}{2}, \phi_{2}=\frac{\alpha-\phi}{2},(0 \leq \phi<2 \pi, \quad 0 \leq \alpha<4 \pi)$.

Then, it is easy to see that $d x_{4}=r d \alpha$ and that by integration over $\alpha$ we are led to $4 \pi \delta_{\nu, 0}$. It follows that the Green's function (104) can be written

$$
G\left(\vec{r}, \overrightarrow{r^{\prime}} ; E\right)=\int_{0}^{\infty} d \tau \exp \left[\frac{i}{\hbar}\left(2 \alpha_{0}+i 0\right) \tau\right] K_{\xi \eta}\left(\phi, \phi^{\prime} ; \tau\right) K\left(\xi, \eta, \xi^{\prime}, \eta^{\prime} ; \tau\right),
$$

where

$$
K_{\xi \eta}\left(\phi, \phi^{\prime} ; \tau\right)=\exp \left\{-\frac{i \tau}{\hbar} \frac{\hbar^{2}}{2 M}\left(\frac{1}{\xi^{2}}+\frac{1}{\eta^{2}}\right)\left[-\frac{\partial^{2}}{\partial \phi^{2}}+F(\tan \phi)\right]\right\} \delta\left(\phi-\phi^{\prime}\right),
$$

and

$$
K\left(\xi, \eta, \xi^{\prime}, \eta^{\prime} ; \tau\right)=\frac{1}{\sqrt{\xi \xi^{\prime} \eta \eta^{\prime}}} \exp \left[-\frac{i \tau}{\hbar} H(\xi, \eta)\right] \delta\left(\xi-\xi^{\prime}\right) \delta\left(\eta-\eta^{\prime}\right),
$$

with

$$
H(\xi, \eta)=-\frac{\hbar^{2}}{2 M}\left(\frac{\partial^{2}}{\partial \xi^{2}}+\frac{\partial^{2}}{\partial \eta^{2}}\right)-\frac{\hbar^{2}}{2 M}\left(k_{1}^{2}-\frac{1}{4}\right)\left(\frac{1}{\xi^{2}}-\frac{1}{\eta^{2}}\right)-4 E\left(\xi^{2}+\eta^{2}\right) .
$$

In order to bring to a constant the mass appearing in the Hamiltonian contained the kernel to expression (115) let's perform the time transformation $\tau \rightarrow \sigma$ defined by $\sigma=\tau\left(\frac{1}{\xi^{2}}+\frac{1}{\eta^{2}}\right)$. Then, it follows that

$$
K_{\xi \eta}\left(\phi, \phi^{\prime} ; \tau\right)=\exp \left\{-\frac{i \sigma}{\hbar} \frac{\hbar^{2}}{2 M}\left[-\frac{\partial^{2}}{\partial \phi^{2}}+F(\tan \phi)\right]\right\} \delta\left(\phi-\phi^{\prime}\right)
$$




$$
=\int d E_{\lambda_{\phi}} \exp \left[-\frac{i}{\hbar}\left(\frac{1}{\xi^{2}}+\frac{1}{\eta^{2}}\right) E_{\lambda_{\phi}} \tau\right] \Psi_{\lambda_{\phi}}(\phi) \Psi_{\lambda_{\phi}}^{*}\left(\phi^{\prime}\right),
$$

with $E_{\lambda_{\phi}}=\frac{\hbar^{2} \lambda_{\phi}^{2}}{2 M}$. Here, we have assumed that the propagator associated with the potential $F(\tan \phi)$ is known.

Let's now insert (118) and (116) in the expression (114), we obtain

$$
\begin{aligned}
G\left(\vec{r}, \overrightarrow{r^{\prime}} ; E\right)= & \int d E_{\lambda_{\phi}} \Psi_{\lambda_{\phi}}(\phi) \Psi_{\lambda_{\phi}}^{*}\left(\phi^{\prime}\right) \frac{1}{\sqrt{\xi \eta \xi^{\prime} \eta^{\prime}}} \int_{0}^{\infty} d \tau \exp \left[\frac{i}{\hbar}\left(2 \alpha_{0}+i 0\right) \tau\right] \\
& \times K\left(\xi, \xi^{\prime} ; \tau\right) K\left(\eta, \eta^{\prime} ; \tau\right),
\end{aligned}
$$

where

$$
\begin{aligned}
K\left(u, u^{\prime} ; \tau\right) & =\exp \left\{-\frac{i \tau}{\hbar}\left[-\frac{\hbar^{2}}{2 M}\left(\frac{\partial^{2}}{\partial u^{2}}-\frac{\lambda_{\phi}^{2} \mp k_{1}^{2}-\frac{1}{4}}{u^{2}}\right)-E u^{2}\right]\right\} \delta\left(u-u^{\prime}\right) \\
& =\exp \left\{-\frac{i \tau}{\hbar}\left[T_{1}(u)+2 \hbar^{2} \omega^{2} T_{3}(u)\right]\right\} \delta\left(u-u^{\prime}\right) ;(u \equiv \xi, \eta),(120)
\end{aligned}
$$

with $\omega=\sqrt{-\frac{2 E}{M}}$.

Following formula (13), the kernels (120) are written

$$
K\left(u, u^{\prime} ; \tau\right)=\frac{M \omega}{i \hbar \sin (\omega \tau)} \exp \left[\frac{i M \omega}{2 \hbar}\left(u^{2}+u^{\prime 2}\right) \cot (\omega \tau)\right] I_{\lambda_{\mp}}\left(\frac{M \omega u u^{\prime}}{i \hbar \sin (\omega \tau)}\right),
$$

with $\lambda_{\mp}=\sqrt{\lambda_{\phi}^{2} \mp k_{1}^{2}}$.

Inserting (121) into (119) and by a procedure similar to that which has led to result (83), we obtain

$$
\begin{aligned}
G\left(\vec{r}, \overrightarrow{r^{\prime}} ; E\right)= & i \hbar \int d E_{\lambda_{\phi}}\left\{\sum_{n_{1}, n_{2}=0}^{\infty} \frac{\Psi_{n_{1}, n_{2}, \lambda_{\phi}}(\xi, \eta, \phi) \Psi_{n_{1}, n_{2}, \lambda_{\phi}}^{*}\left(\xi^{\prime}, \eta^{\prime}, \phi^{\prime}\right)}{E+i 0-E_{N}}\right. \\
& \left.+\int_{0}^{\infty} d p \int_{-\infty}^{\infty} d \varsigma \frac{\Psi_{p, \varsigma, \lambda_{\phi}}(\xi, \eta, \phi) \Psi_{p, \varsigma, \lambda_{\phi}}^{*}\left(\xi^{\prime}, \eta^{\prime}, \phi^{\prime}\right)}{E+i 0-E_{p}}\right\},
\end{aligned}
$$

with the normalized wave functions and the energy spectrum for 
-the bound states:

$$
\begin{aligned}
& \Psi_{n_{1}, n_{2}, \lambda_{\phi}}(\xi, \eta, \phi)= {\left[\frac{2}{a^{3} N^{4}} \frac{n_{1} ! n_{2} !}{\Gamma\left(n_{1}+\lambda_{-}+1\right) \Gamma\left(n_{2}+\lambda_{+}+1\right)}\right]^{\frac{1}{2}}\left(\frac{\xi}{a N}\right)^{\frac{\lambda_{-}}{2}} } \\
& \times\left(\frac{\eta}{a N}\right)^{\frac{\lambda_{+}}{2}} \exp \left(-\frac{\xi^{2}+\eta^{2}}{2 a N}\right) L_{n_{1}}^{\lambda_{-}}\left(\frac{\xi^{2}}{a N}\right) L_{n_{2}}^{\lambda_{+}}\left(\frac{\eta^{2}}{a N}\right) \\
& \times \Psi_{\lambda_{\phi}}(\phi), \\
& E_{N}=-\frac{M \alpha_{0}^{2}}{2 \hbar^{2} N^{2}}, \quad N=n_{1}+n_{2}+\frac{1}{2}\left(\lambda_{-}+\lambda_{+}\right)+1
\end{aligned}
$$

-the continuous states:

$$
\begin{aligned}
\Psi_{p, \varsigma, \lambda_{\phi}}(\xi, \eta, \phi)= & \frac{\left|\Gamma\left(\frac{1}{2}\left(1+\lambda_{+}\right)+\frac{i}{2 p}\left(\frac{1}{a}+\varsigma\right)\right) \Gamma\left(\frac{1}{2}\left(1+\lambda_{-}\right)+\frac{i}{2 p}\left(\frac{1}{a}-\varsigma\right)\right)\right|}{2 \pi \Gamma\left(1+\lambda_{+}\right) \Gamma\left(1+\lambda_{-}\right)} \\
& \times \frac{e^{\frac{\pi}{2 a p}}}{\sqrt{p} \xi \eta} M_{-\frac{i}{2 p}\left(\frac{1}{a}+\varsigma\right), \frac{\lambda_{-}}{2}}\left(-i p \xi^{2}\right) M_{-\frac{i}{2 p}\left(\frac{1}{a}-\varsigma\right), \frac{\lambda_{+}}{2}}\left(-i p \eta^{2}\right) \\
& \times \Psi_{\lambda_{\phi}}(\phi) .
\end{aligned}
$$

\section{Spherical coordinates}

With the help of the change of variables defined by

$$
(\xi, \eta) \rightarrow\left(\sqrt{2 r} \cos \frac{\theta}{2}, \sqrt{2 r} \sin \frac{\theta}{2}\right)
$$

and by applying the Bateman 's expansion formula (26), expression (119) is rewritten

$$
\begin{aligned}
G\left(\vec{r}, \overrightarrow{r^{\prime}} ; E\right)= & \int d E_{\lambda_{\phi}} \Psi_{\lambda_{\phi}}(\phi) \Psi_{\lambda_{\phi}}^{*}\left(\phi^{\prime}\right) \sum_{n=0}^{\infty} \Phi_{n}^{\left(\lambda_{+}, \lambda_{-}\right)}\left(\frac{\theta}{2}\right) \\
& \times \Phi_{n}^{\left(\lambda_{+}, \lambda_{-}\right)}\left(\frac{\theta^{\prime}}{2}\right) \frac{1}{\sqrt{\sin \theta \sin \theta^{\prime}}} \int_{0}^{\infty} d \tau \frac{M \omega}{i \hbar \sin (\omega \tau)}
\end{aligned}
$$




$$
\begin{aligned}
& \times \exp \left[\frac{i}{\hbar}\left(2 \alpha_{0}+i 0\right) \tau\right] \exp \left[\frac{i M \omega}{\hbar}\left(r+r^{\prime}\right) \cot (\omega \tau)\right] \\
& \times I_{2 n+\lambda_{+}+\lambda_{-}+1}\left(\frac{2 M \omega \sqrt{r r^{\prime}}}{i \hbar \sin (\omega \tau)}\right) .
\end{aligned}
$$

Performing the integration over the time variable $\tau$ with the help of formula (30), we obtain the following final form

$$
\begin{aligned}
G\left(\vec{r}, \overrightarrow{r^{\prime}} ; E\right)= & \int d E_{\lambda_{\phi}} \Psi_{\lambda_{\phi}}(\phi) \Psi_{\lambda_{\phi}}^{*}\left(\phi^{\prime}\right) \sum_{n=0}^{\infty} \frac{1}{\sqrt{\sin \theta \sin \theta^{\prime}}} \Phi_{n}^{\left(\lambda_{+}, \lambda_{-}\right)}\left(\frac{\theta}{2}\right) \\
& \times \Phi_{n}^{\left(\lambda_{+}, \lambda_{-}\right)}\left(\frac{\theta^{\prime}}{2}\right) \frac{1}{2 i \omega r r^{\prime}} \frac{\Gamma\left(p+n+\frac{1}{2}\left(\lambda_{+}+\lambda_{-}\right)+1\right)}{\Gamma\left(2 n+\lambda_{+}+\lambda_{-}+2\right)} \\
& \times M_{-p, n+\frac{1}{2}\left(\lambda_{+}+\lambda_{-}+1\right)}\left(\frac{2 M \omega}{\hbar} r^{\prime}\right) \\
& \times W_{-p, n+\frac{1}{2}\left(\lambda_{+}+\lambda_{-}+1\right)}\left(\frac{2 M \omega}{\hbar} r\right),
\end{aligned}
$$

where $r>r^{\prime}$ and $p=-\frac{\alpha_{0}}{\hbar \omega}$.

Following the calculation procedure in subsection (IV.2), one shows that

$$
\begin{aligned}
G\left(\vec{r}, \overrightarrow{r^{\prime}} ; E\right)= & i \hbar \int d E_{\lambda_{\phi}} \sum_{n=0}^{\infty}\left\{\sum_{l=0}^{\infty} \frac{\Psi_{l, n, \lambda_{\phi}}(r, \theta, \phi) \Psi_{l, n, \lambda_{\phi}}^{*}\left(r^{\prime}, \theta^{\prime}, \phi^{\prime}\right)}{E+i 0-E_{N}}\right. \\
& \left.+\int_{0}^{\infty} d k \frac{\Psi_{k, n, \lambda_{\phi}}(r, \theta, \phi) \Psi_{k, n, \lambda_{\phi}}^{*}\left(r^{\prime}, \theta^{\prime}, \phi^{\prime}\right)}{E+i 0-\frac{\hbar^{2} k^{2}}{2 M}}\right\},
\end{aligned}
$$

where the normalized wave functions and the energy spectrum are given by

$$
\begin{aligned}
\Psi_{l, n, \lambda_{\phi}}(\vec{r})= & \frac{1}{a\left(l+\lambda_{1}+\frac{1}{2}\right)^{2}}\left[\frac{2 l !}{a \Gamma\left(l+2 \lambda_{1}+1\right)}\right]^{\frac{1}{2}}\left(\frac{2 r}{a\left(l+\lambda_{1}+\frac{1}{2}\right)}\right)^{\lambda_{1}-\frac{1}{2}} \\
& \times \exp \left(-\frac{r}{a\left(l+\lambda_{1}+\frac{1}{2}\right)}\right) L_{l}^{2 \lambda_{1}}\left(\frac{2 r}{a\left(l+\lambda_{1}+\frac{1}{2}\right)}\right) \sqrt{\frac{2}{\sin \theta}} \\
& \times \Phi_{n}^{\left(\lambda_{+}, \lambda_{-}\right)}\left(\frac{\theta}{2}\right) \Psi_{\lambda_{\phi}}(\phi),
\end{aligned}
$$




$$
E_{N}=-\frac{M \alpha_{0}^{2}}{2 \hbar^{2} N^{2}}, \quad N=l+\lambda_{1}+\frac{1}{2} \quad \text { and } \quad \lambda_{1}=n+\frac{1}{2}\left(\lambda_{+}+\lambda_{-}+1\right),
$$

for the bound states and

$$
\begin{aligned}
& \Psi_{k, n, \lambda_{\phi}}(\vec{r})= \frac{\left|\Gamma\left(\frac{1}{2}+\lambda_{1}-\frac{i}{a k}\right)\right|}{\Gamma\left(2 \lambda_{1}+1\right)} \frac{e^{\frac{\pi}{2 a k}}}{r} M_{-\frac{i}{a k}, \lambda_{1}}(-2 i k r) \frac{1}{\sqrt{2 \sin \theta}} \Phi_{n}^{\left(\lambda_{+}, \lambda_{-}\right)}\left(\frac{\theta}{2}\right) \\
& \times \Psi_{\lambda_{\phi}}(\phi), \\
& E_{k}=\frac{\hbar^{2} k^{2}}{2 M},
\end{aligned}
$$

for the continuous states.

\section{Conclusion}

In this paper, we have analyzed through the Milshtein and Strakhovenko variant of the so $(2,1)$ Lie algebra a set of potentials belonging to three different classes of Smorodinsky-Winternitz potentials. The use of the second order differential operators of this algebra allows to write the Hamiltonian of these physical systems in form of a linear combination of the latter. Using the Schwinger's integral representation and with the help of two BakerCampbell-Hausdorff formulas allowing the separation of the $T_{i}$ operators and thus simplifying their action on a Laplace transform of a well chosen Dirac distribution, we have shown that we can construct the Green's functions in compact form in different coordinate systems. This method can be compared to the approach of the Schrödinger equation and to the Feynman's path integral technique. It gives a local view of the problem under consideration like the Schrödinger approach, but its advantage is in the computation of the explicit and compact form of the Green's function from which the energy spectrum and the suitably normalized wave functions are simultaneously extracted for the bound states and for the continuous states if they exist at one and the same time.

The advantage of the path integral approach in comparison with the algebraic method is that it provides a global view of the dynamics of the physical system, but a problem of singularity is often to be found at the origin of coordinates, and it requires a regularization which is rather complicated to perform. From this point of view, we can assert that this algebraic method 
has the advantage of not presenting this problem owing to the fact that it is local.

The method of Milshtein and Strakhovenko could become a powerful alternative approach to the path integral technique if we manage to extend its use to the treatment of Pöschl-Teller potential class. 


\section{References}

${ }^{1}$ H. Kleinert, Fortschr. Phys. 6, 1 (1968); Group Dynamics of the Hydrogen ( Lectures presented at the1967 Boulder Summer School, published in Lectures in Theoretical Physics, Vol.XB, A. O. Barut and W. E. Brittin, eds. Gordon and Breach, New York, 1968 ).

${ }^{2}$ G. A. Natanzon, Teor. Mat. Fiz. 38, 146 (1979).

${ }^{3}$ M. J. Englefield, Group Theory and the Coulomb Problem ( Wiley, New York, 1972 ).

${ }^{4}$ J. N. Huffaker and P. H. Dwivedi, J. Math. Phys. 16, 862 (1975).

${ }^{5}$ J. Cizek and J. Paldus, Int. J. Quantum Chem. 12, 875 (1977).

${ }^{6}$ M. Berrondo and A. Palma, J. Phys. A: Math Gen. 13, 7 (1980).

${ }^{7}$ J. Wu and Y. Alhassid, J. Math. Phys. 31, 557 (1990).

${ }^{8}$ P. Cordero and S. Salamo, J. Phys. A: Math. Gen. 24, 5299 (1991); Foun. Phys. 23, 675 (1993).

${ }^{9}$ I. L. Cooper, J. Phys. A: Math. Gen. 26, 1601 (1993).

${ }^{10}$ C. C. Gerry, Phys. Lett. A 118, 445 (1986).

${ }^{11}$ H. Hartmann, Theor. Chem. Acta 24, 201 (1972).

${ }^{12}$ M. V. Carpio-Bernido and C. C. Bernido, Phys. Lett. A 137, 1 (1989).

${ }^{13}$ C. Quesne, J. Phys. A: Math. Gen. 21, 3039 (1988).

${ }^{14}$ A. I. Milshtein and V. M. Strakhovenko, Phys. Lett. A 90, 447 (1982).

${ }^{15}$ L. Chetouani, L. Guechi and T.F. Hammann, Helv. Phys. Acta 65, 1069 (1992); Czech. J. Phys. 42, 913 (1992).

${ }^{16}$ A. N. Vaidya and H. Boschi-Filho, J. Math. Phys. 31, 1951 (1990).

${ }^{17}$ H. Boschi-Filho and A. N. Vaidya, Ann. Phys., (NY) 212, 1 (1991). 
${ }^{18}$ H. Boschi-Filho, M. de Souza and A. N. Vaidya, J. Phys. A: Math. Gen. 24, 4981 (1991).

${ }^{19}$ L. Chetouani, L. Guechi, T. F. Hammann and A. Lecheheb, J. Math. Phys. 36, 1016 (1995).

${ }^{20}$ L. Chetouani, L. Guechi and T. F. Hammann, J. Math. Phys. 33, 3410 (1992).

${ }^{21}$ H. Boschi-Filho and A. N. Vaidya, Phys. Lett. A 149, 336 (1990).

${ }^{22}$ L. Chetouani, L. Guechi, A. Lecheheb and T. F. Hammann, Helv. Phys. Acta 68, 158 (1995).

${ }^{23}$ A. A. Makarov, J. A. Smorodinsky, Kh. Valiev and P. Winternitz, Nuovo Cimento A 52, 1061 (1967).

${ }^{24}$ M. Kibler and P. Winternitz, J. Phys. A: Math. Gen.20, 4097 (1987); Phys. Lett. A 147, 338 (1990).

${ }^{25}$ N. W. Evans, Phys. Lett. A 147, 483 (1990).

${ }^{26}$ N. W. Evans, J. Math. Phys. 31, 600 (1990); ibid 32, 3369 (1991).

${ }^{27}$ D. Bonatos, C. Daskaloyannis and K. Kokkotas, Phys. Rev. A 48, R 3407 (1993); J. Hietarinta, Phys. Rep. 147, 87 (1987).

${ }^{28}$ N. W. Evans, Phys. Rev. A 41, 5666 (1990).

${ }^{29}$ C. Grosche, G. S. Pogosyan and A. N. Sissakian, Fortschr. Phys. 43, 453 (1995).

${ }^{30} \mathrm{H}$. Kleinert, Path Integrals in Quantum Mechanics, Statistics and Polymer Physics ( World Scientific, Singapore, 1990 ).

${ }^{31}$ R. Gilmore, Lie Groups, Lie Algebras and Some of Their Applications ( Wiley, New York, 1974 ).

${ }^{32}$ J. Schwinger, Phys. Rev. 82, 664 (1951).

${ }^{33}$ B. G. Wybourne, Classical Groups for Physicists (Wiley, New York 1974). 
${ }^{34}$ I. H. Duru and H. Kleinert, Fortschr. Phys. 30, 401 (1982).

${ }^{35}$ A. Inomata, Phys. Lett. A 87, 387 (1982).

${ }^{36}$ T. Levita Civita, Opere Matematiche, V., 2 (1956).

${ }^{37}$ I. S. Gradshtein and I. M. Ryzhik, Table of Integrals, Series and Products ( Academic, New York, 1965 ) p. 1038, Eq. (8.976).

${ }^{38}$ A. Erdelyi, W. Magus, F. Oberhettinger and F. G. Tricomi, Higher Transcendental Functions ( Mc Graw-Hill, New York, 1953 ) Vol. 2.

${ }^{39}$ P. M. Morse and H. Feshbach, Methods of Theoretical Physics ( Mc GrawHill, New York, 1953 ) Vol. 1, p. 781.

${ }^{40}$ H. Buchholz, The Confluent Hypergeometric Function, Springer Tracts in Natural Philosophy ( Springer-Verlag, Berlin, 1969 ) vol.15.

${ }^{41}$ I. H. Duru and H. Kleinert, Phys. Lett. B 84, 185 (1979).

${ }^{42}$ R. Ho and A. Inomata, Phys. Rev. Lett. 48, 231 (1982).

${ }^{43}$ K. Pak and I. Sökmen, Phys. Lett. A 100, 327 (1984).

${ }^{44}$ A. Inomata, Phys. Lett. A 101, 253 (1984).

${ }^{45}$ F. Steiner, Phys. Lett. A 106, 363 (1984).

${ }^{46}$ L. Chetouani and T. F. Hammann, J. Math. Phys. 27, 2944 (1986).

${ }^{47}$ L. Chetouani and T. F. Hammann, Nuovo Cimento B 98, 1 (1987).

${ }^{48}$ H. Kleinert, Phys. Lett. A 120, 361 (1987).

${ }^{49}$ D. P. L. Castrigiano and F. Stärk, J. Math. Phys. 30, 2785 (1989).

${ }^{50}$ S. N. Storchak, Theor. Math. Phys. 82, 32 (1990).

${ }^{51}$ C. Grosche, Fortschr. Phys. 40, 695 (1992) .

${ }^{52}$ M. V. Carpio-Bernido, J. Math. Phys. 32, 1799 (1991).

${ }^{53}$ M. Kibler and C. Campigotto, Int. J. Quantum Chem. 45, 209 (1993). 
${ }^{54}$ M. Kibler, L. G. Mardoyan and G. S. Pogosyan, Int. J. Quantum Chem. 52, 1301 (1994).

${ }^{55}$ W. Thirring, Classical Field Theory ( Springer-Verlag, New York, 1986 ) p. 140.

${ }^{56} \mathrm{M}$. V. Carpio-Bernido, C. C. Bernido and A. Inomata, in Third International Conference on Path Integrals from meV to MeV, 1989, p. 442, Eds. V. Sa-Yakanit et al ( World Scientific, Singapore, 1989 ).

${ }^{57}$ M. V. Carpio-Bernido and A. Inomata, in Bielefeld Encounters in Physics and Mathematics VII,Path Integrals from meV to MeV, 1985, p.261, Eds. M. C. Gutzwiller, A. Inomata, J. R. Klauder and L. Streit (World Scientific, Singapore, 1986 ).

${ }^{58}$ I. Sökmen, Phys. Lett. A 115, 249 (1986).

${ }^{59}$ L. Chetouani, L. Guechi and T. F. Hammann, Phys. Lett. A 125, 277 (1987).

${ }^{60}$ M. V. Carpio-Bernido, J. Phys. A: Math. Gen. 24, 3013 (1991).

${ }^{61}$ M. Kibler and C. Campigotto, Phys. Lett. A 181, 1 (1993).

${ }^{62}$ M. Kibler, G. H. Lamot and P. Winternitz, Int.J. Quantum Chem. 43, 625 (1992).

${ }^{63}$ F. Calogero, J. Math. Phys. 10, 2191 (1969).

${ }^{64}$ M. Kibler and T. Negadi, Int. J. Quantum Chem. 26, 405 (1984).

${ }^{65}$ A. Guha and S. Mukherjee, J. Math. Phys. 28, 840 (1987).

${ }^{66}$ Ya. A. Granovsky, A. S. Zhedanov and I. M. Lutzenko, J. Phys. A: Math. Gen. 24, 3887 (1991).

${ }^{67}$ A. S. Zhedanov, J. Phys. A: Math. Gen. 26, 4633 (1993).

${ }^{68}$ M. Kibler, L. G. Mardoyan and G. S. Pogosyan, Int. J. Quantum Chem. 52, 1301 (1994). 
${ }^{69}$ Y. Aharonov and D. Bohm, Phys. Rev. 115, 485 (1959).

${ }^{70}$ L. Chetouani, L. Guechi and T. F. Hammann, J. Math. Phys. 30, 655 (1989).

${ }^{71}$ I. Sökmen, Phys. Lett. A 132, 65 (1988).

${ }^{72}$ Le Van Hoang and N. T. Giang, J. Phys. A : Math. Gen. 26, 3333 (1993).

${ }^{73}$ M. Kibler and T. Negadi, Phys. Lett. A 124, 42 (1987).

${ }^{74}$ P. Kustaanheimo and E. Stiefel, J. Reine Angew. Math. 218, 204 (1965). 